Preprint typeset in JHEP style - HYPER VERSION

\title{
Exploring novel correlations in trilepton channels at the LHC for the minimal supersymmetric inverse seesaw model
}

\author{
Subhadeep Mondal, ${ }^{a}$ Sanjoy Biswas, ${ }^{b}$ Pradipta Ghosh ${ }^{c}$ and Sourov Roy ${ }^{a}$ \\ ${ }^{a}$ Department of Theoretical Physics, \\ Indian Association for the Cultivation of Science, \\ $2 A$ \& $2 B$ Raja S.C. Mullick Road, Kolkata 700032, India \\ ${ }^{b}$ INFN, Sezione di Roma, Dipartimento di Fisica, Università di Roma La Sapienza \\ Piazzale Aldo Moro 2; I-00185 Rome, Italy \\ ${ }^{c}$ Departamento de Física Teórica UAM and Instituto de Física Teórica UAM/CSIC, \\ Universidad Autónoma de Madrid (UAM), Cantoblanco, 28049 Madrid, Spain \\ E-mails tpsm2@iacs.res.in, Sanjoy.Biswas@roma1.infn.it, \\ pradipta.ghosh@uam.es, tpsr@iacs.res.in
}

\begin{abstract}
We investigate signatures of the minimal supersymmetric inverse seesaw model at the large hadron collider (LHC) with three isolated leptons and large missing energy $\left(3 \ell+\mathbb{E}_{T}\right.$ or $2 \ell+1 \tau+\mathbb{E}_{T}$, with $\left.\ell=e, \mu\right)$ in the final state. This signal has its origin in the decay of chargino-neutralino $\left(\widetilde{\chi}_{1}^{ \pm} \widetilde{\chi}_{2}^{0}\right)$ pair, produced in $p p$ collisions. The two body decays of the lighter chargino into a charged lepton and a singlet sneutrino has a characteristic decay pattern, which is correlated with the observed large atmospheric neutrino mixing angle. This correlation is potentially observable at the LHC by looking at the ratios of cross sections of the trilepton $+\mathbb{E}_{T}$ channels in certain flavour specific modes. We show that even after considering possible leading standard model backgrounds these final states can lead to reasonable discovery significance at the LHC with both $7 \mathrm{TeV}$ and $14 \mathrm{TeV}$ center-of-mass energy.
\end{abstract}

Keywords: Beyond Standard Model, Supersymmetric Standard Model, Neutring Physics. 


\section{Contents}

1. Introduction

2. Model 田

3. Decays of chargino and neutralino 6

3.1 Chargino decay 6

3.2 Neutralino decay 8

3.3 Trilepton signal and the benchmark points 9

4. Event generation and

background analysis $\quad 11$

5. Results 14

6. Conclusion 21

\section{Introduction}

Supersymmetry is one of the most well-motivated theories for explaining new physics beyond the standard model (SM) of particle physics. With the initiation of the large hadron collider (LHC) experiment at CERN, discovery of weak scale supersymmetric particles is highly envisaged. Moreover, the stable, neutral, and weakly interacting lightest supersymmetric particle (LSP) in an R-parity conserving theory can be an ideal candidate for a non-baryonic dark matter for explaining the data from the WMAP satellite and large scale structure formation.

On the other hand, experimental evidences of neutrino oscillation have firmly established the necessity of massive neutrinos and the associated non-trivial mixing in the leptonic sector (see references [1-3] for the latest update on the three flavour global neutrino data). This is also a definite and very important indication of new physics because non-zero neutrino masses and mixing are not included in the SM.

Seesaw mechanism [4-10] turns out to be the most simple and natural $[11,12]$ way to accommodate tiny neutrino masses, $m_{\nu_{i}}\left(\sum m_{\nu_{i}}<0.58 \mathrm{eV}\right.$ [13]) both for supersymmetric and non-supersymmetric theories. However, neutrino mass models with canonical seesaw mechanism cannot be tested directly since the associated heavy states (to ensure smallness in neutrino masses) are usually $\sim \mathcal{O}\left(10^{15} \mathrm{GeV}\right)$ and thus well beyond the reach of any ongoing collider experiment.

The inverse seesaw [14-16] is one of the viable alternatives with a seesaw mechanism operational at the $\mathrm{TeV}$ scale, which is well within the reach of the LHC. By proposal inverse 
seesaw mechanism relies on a new mass parameter, which breaks lepton number explicitly by two units, and at the same time small enough to ensure small neutrino masses without the requirement of any super heavy states. The smallness of this new lepton number breaking scale can arise either spontaneously [16] or dynamically [17].

In the supersymmetric inverse seesaw model, $R$-parity [18-21] is conserved as a consequence of lepton number violation by two units. Thus the LSP is stable for this class of models and can be a viable candidate for the cold dark matter of the Universe.

In this paper, we study signatures of the minimal supersymmetric version of the inverse seesaw model at the LHC. This model can accommodate three flavour global neutrino data $[1,2]$ with a pair of SM gauge singlet superfields, which carry lepton number. These singlet scalars (having mixing with the doublet sneutrinos) can be thermal cold dark matter candidates, because the neutrino Yukawa coupling in this model is $\sim \mathcal{O}\left(10^{-1}\right)$. In a supersymmetric inverse seesaw model just one pair of singlets can explain the observed neutrino experimental data [22]. On the other hand, a non-supersymmetric inverse seesaw model requires at least two pairs of singlets [23]. Apart from the neutrino masses and mixing, the supersymmetric inverse seesaw model has also been analysed earlier in the context of lepton flavour violation [22,24-27], leptogenesis [28-30], dark matter [31-33] etc.

In the case when one of the singlet sneutrinos is the LSP, the phenomenology at the LHC can be very interesting. This is because in this model the neutrino Yukawa coupling is large and can lead to lepton flavor violating (LFV) coupling of the sneutrinos with a charged lepton and the chargino. These couplings are related to the observed neutrino mixing angles and hence by studying the collider signatures of this model it is possible to study the relation between neutrino physics and the physics at the high energy colliders.

Supersymmetric particle searches from the $1 \mathrm{fb}^{-1}$ data, collected by ATLAS and CMS for $p p$ collision at center-of-mass energy, $\sqrt{s}=7 \mathrm{TeV}$, has found no significant signal over the expected SM background. In the context of the constrained minimal supersymmetric standard model (CMSSM), searches by ATLAS exclude squarks and gluinos with masses below $950 \mathrm{GeV}$ [34] at 95\% C.L. for some particular choice of other parameters. The results from CMS extend the mass limit to $1.1 \mathrm{TeV}$ [35-37]. However, the third generation squarks can still be somewhat lighter, particularly in the context of a more general MSSM scenario. This is the reason, in this work, we choose to work with a spectrum where the squarks of the first two generations and the gluinos are very heavy $(\sim 1 \mathrm{TeV})$ and the electroweak sector is relatively light so that the lighter chargino and neutralinos can be produced at the LHC. If in the production or in the decay chain the lighter chargino $\left(\tilde{\chi}_{1}^{ \pm}\right)$appears then it can have a decay into a charged lepton $(l)$ via $\tilde{\chi}_{1}^{ \pm} \rightarrow l^{ \pm}+\tilde{N}$, where $\tilde{N}$ represents the singlet sneutrino LSP. The ratios of the decay branching ratios into different charged lepton flavors can be shown to correlate with the neutrino mixing angles [22]. Our aim in this paper is to look at these correlations by studying the trilepton $+\mathbb{E}_{T}$ signature from the associated production of the lighter chargino $\left(\tilde{\chi}_{1}^{ \pm}\right)$and the second lightest neutralino $\left(\tilde{\chi}_{2}^{0}\right)$ at the LHC.

Similar correlations also appear in the decay of the LSP in the model of bilinear Rparity violation [38-45], spontaneous R-parity violation [46] and in $\mu \nu \mathrm{SSM}$ [47-49]. In these models correlations with neutrino mixing angles have been studied in various context 
in the case of a neutralino LSP decays as well as for other LSPs including the chargino [44]. The final states discussed in these cases generally include multi - leptons + jets $+\mathbb{E}_{T}$ along with the presence of displaced vertices originating from the long-lived LSP. In the present paper we have studied this correlation in the decay of the NLSP chargino in minimal supersymmetric inverse seesaw model (MSISM) through the cleaner trilepton $+\mathbb{E}_{T}$ final state in the absence of any displaced vertex.

It is known that at least two non-vanishing neutrino masses are essential [50-52] to account for oscillation data $[1,2]$. In the minimal supersymmetric inverse seesaw model with one pair of singlets only one neutrino mass is generated at the tree level, while another neutrino mass is originating from the sneutrino-anti-sneutrino loop [53,54]. This feature is analogous to the $R$-parity violating (see review [55] and references therein) models of light neutrino mass generation with bilinear terms [54,56-64]. Issues of neutrino mass generation in MSISM have been addressed in ref. [22]. Similar issues have been discussed in refs. [24,31], but with three generations of singlets. A model of neutrino mass generation where the origin of neutrino mass is radiative and suppressed by inverse seesaw scale has been advocated in ref. [65].

In MSISM, the atmospheric neutrino mixing angle $\left(\theta_{23}\right)$ correlates not only with the ratio of the branching ratios of the lighter chargino $\left(\widetilde{\chi}_{1}^{ \pm}\right)$decay modes but also with ratio of the branching ratios of lepton flavour violating decays, $\tau / \mu \rightarrow \ell+\gamma[22]$. On the other hand, trilepton signals ( $3 l$, with or without tau lepton(s)) have been extensively studied for a long time as an important probe for supersymmetric models [66-77] (see also references $[3,4,6,7]$ of ref. [68]). Besides, a hadronically quiet event like this always has the favour of reducible backgrounds. Moreover, multi-lepton signals have already been considered as an important probe for seesaw models [78-80]. Being motivated by these features together with the novel correlations mentioned earlier, we aim to perform a detailed analysis of trilepton $(3 \ell, 2 \ell+1 \tau)+\mathbb{E}_{T}$ signals for the MSISM taking into account possible SM backgrounds.

As mentioned earlier, we search for the trilepton signatures, arising from the decay of $\widetilde{\chi}_{2}^{0} \widetilde{\chi}_{1}^{ \pm}$pair. In our chosen parameter points the associated production of the lightest chargino with the next-to-lightest neutralino, $p p \rightarrow \widetilde{\chi}_{2}^{0} \widetilde{\chi}_{1}^{ \pm}+X$ can occur with a detectable rate at the LHC. In addition, the lighter chargino and the second lightest neutralino decays via two body leptonic modes with large branching ratios. The final state signal will produce three charged lepton and missing energy signature $\left(3 \ell+\mathbb{E}_{T}\right.$ or $\left.2 \ell+1 \tau+\mathbb{E}_{T}\right)$, because of the presence of the stable singlet sneutrino LSP. It is interesting to note that in our analysis the lightest neutralino $\widetilde{\chi}_{1}^{0}$ decays into a singlet scalar LSP and a light neutrino. Both of these decay products escape detection and thus $\widetilde{\chi}_{1}^{0}$ can be thought of as a virtual LSP, which also yields the missing energy signature at an accelerator experiment similar to that by an LSP. We investigate three body final states like $3 \ell(\ell=e, \mu)$ and also $2 \ell+\tau$-jet. Final states with more than one tau lepton have been dropped for small $\tau$ detection efficiency [81]. In the course of present analysis we choose to work with non-universal gaugino masses but maintain $M_{2}>M_{1}$, where $M_{1}\left(M_{2}\right)$ are the soft masses for $U(1)(S U(2))$ gaugino(s). It has also been assumed that $\mu>M_{2}$, where $\mu$ is the coefficient of the only bilinear term in the superpotential of the minimal supersymmetric standard model (MSSM). With 
such a choice, $\widetilde{\chi}_{2}^{0}, \widetilde{\chi}_{1}^{ \pm}$are essentially gaugino like. Moreover, since the first two generation squark masses are heavy, the process $p p \rightarrow \widetilde{\chi}_{2}^{0} \widetilde{\chi}_{1}^{ \pm}$receives prime contributions from $W^{ \pm}$ boson mediated processes. Three of our benchmark points (BP1, BP3 and BP4 as defined later) are chosen with this criteria. We have, however, also considered the situation when $M_{2}>\mu$ for another benchmark point (BP2). However, in a situation like this, $\widetilde{\chi}_{2}^{0}, \widetilde{\chi}_{1}^{ \pm}$are higgsino like and consequently yield a smaller cross section for the process $p p \rightarrow \widetilde{\chi}_{2}^{0} \widetilde{\chi}_{1}^{ \pm}$. We show later that in a scenario like this the trileptonic final state possesses lower significance compared to the $\mu>M_{2}$ scenario. We will discuss this issue in more details later in section 5. Having heavy squarks is also useful for suppressing flavour violating processes in the quark sector.

The ratio of the branching ratios for $\tilde{\chi}_{1}^{ \pm}$decaying into $\mu$ and $\tau$ channel in MSISM shows sharp correlation with $\tan ^{2} \theta_{23}$ [22]. It is clear that one of the three charged leptons appearing either in $3 \ell+\mathbb{E}_{T}$ or $2 \ell+\tau+\mathbb{E}_{T}$ final states must have its origin in $\widetilde{\chi}_{1}^{ \pm}$decay. Using this idea we find that the ratio $\frac{\sigma\left(\mu^{ \pm}+\sum \ell \ell\right)}{\sigma\left(\tau^{ \pm}+\sum \ell \ell\right)}$, with $\ell=e, \mu$ shows nice correlation with $\tan ^{2} \theta_{23}$ even after the application of different kinematical cuts to reduce SM backgrounds. Definitely, that $\mu$ and $\tau$ are coming from lightest chargino decay. Existence of this final state correlation with neutrino mixing angle along with a large amount of $\mathbb{E}_{T}$ provides a distinct signature for the MSISM.

The present paper is organized as follows. We start with a brief description of the underlying model in section 2 and discuss the generation of small neutrino masses as well as masses and mixing in the sneutrino sector. In section 3 we discuss the decay modes of the lighter chargino and the lighter neutralinos $\left(\widetilde{\chi}_{1}^{0}, \widetilde{\chi}_{2}^{0}\right)$ in our model and present a set of four benchmark points (BPs) studied in this work. In addition we discuss how these decay modes can lead to the trilepton final states at the LHC. Section 1 discusses the details of the signal event generation and the background analysis. We present our results in section 5 and finally, our conclusions are provided in section 6 .

\section{Model}

In the MSISM the particle content of the MSSM is extended by a pair of SM singlet fields, $\widehat{\nu}^{c}$ and $\widehat{S}$ having lepton numbers -1 and +1 , respectively. The model superpotential following refs. $[22,31]$ is written as

$$
W=W_{M S S M}+\varepsilon_{a b} h_{\nu}^{i} \widehat{L}_{i}^{a} \widehat{\nu}^{c} \widehat{H}_{u}^{b}+M_{R} \widehat{\nu}^{c} \widehat{S}+\frac{1}{2} \mu_{S} \widehat{S} \widehat{S}
$$

where $W_{M S S M}$ is the MSSM superpotential. In eq. (2.1) $\hat{L}_{i}$ s are the $S U(2)_{L}$ doublet lepton superfields and $\hat{H}_{u}$ represents a up-type Higgs superfield. $\hat{\nu}^{c}$ represents a right handed neutrino superfield whereas $\hat{S}$ is another SM gauge-singlet superfield, but with non-zero lepton number. In eq. (2.1) coefficient of the lepton number violating term is given by $\mu_{S}$. In the limit $\mu_{S} \rightarrow 0$, MSISM superpotential (see eq. (2.1)) restores lepton number conservation, which is consistent with the t'Hooft naturalness criteria [82].

The corresponding soft supersymmetry breaking Lagrangian is given by

$$
-\mathcal{L}_{\mathrm{soft}}=-\mathcal{L}_{\mathrm{soft}}^{\mathrm{MSSM}}+m_{\nu^{c}}^{2} \widetilde{\nu}^{c \dagger} \widetilde{\nu}^{c}+m_{S}^{2} \widetilde{S}^{\dagger} \widetilde{S}
$$




$$
+\left(\varepsilon_{a b} A_{h_{\nu}}^{i} \widetilde{L}_{i}^{a} \widetilde{\nu}^{c} H_{u}^{b}+B_{M_{R}} \widetilde{\nu}^{c} \widetilde{S}+\frac{1}{2} B_{\mu_{S}} \widetilde{S} \widetilde{S}+\text { h.c. }\right)
$$

with $-\mathcal{L}_{\text {soft }}^{\text {MSSM }}$ representing soft terms of the MSSM. Just like the coefficient $\mu_{S}$ appearing in the superpotential (eq. (2.1)), the $B_{\mu_{S}}$ parameter in the soft terms (eq. (2.2)) violates lepton number by two units. The MSISM thus includes two lepton number violating parameters. Both of these will contribute to the Majorana neutrino mass matrix [22].

Tree level neutrino mass matrix in the MSISM is a $5 \times 5$ matrix in the basis $\left(\nu_{l}, \nu^{c}, S\right)$, where, $l \equiv e, \mu, \tau$. This is given by

$$
\left(\begin{array}{ccccc}
0 & 0 & 0 & m_{D_{1}} & 0 \\
0 & 0 & 0 & m_{D_{2}} & 0 \\
0 & 0 & 0 & m_{D_{3}} & 0 \\
m_{D_{1}} & m_{D_{2}} & m_{D_{3}} & 0 & M_{R} \\
0 & 0 & 0 & M_{R} & \mu_{s}
\end{array}\right)
$$

where $m_{D_{i}} \equiv h_{\nu}^{i} v_{u}(i=1,2,3)$ are the three light neutrino Dirac masses with $v_{u}$ as the vacuum expectation value of the up-type Higgs field, $\left\langle H_{u}^{0}\right\rangle$. The quantities $h_{\nu}^{i}$ are the neutrino Yukawa couplings. The structure of the matrix shown in eq. (2.3) can be readily understood from eq. (2.1) by looking at the mixing between different doublet and singlet neutral fermions. The effective $3 \times 3$ mass matrix for the light neutrinos can be obtained as

$$
\left(M_{\text {tree }}^{\nu}\right)_{i j}=\frac{\mu_{S}}{\left(M_{R}^{2}+\sum m_{D_{k}}^{2}\right)} m_{D_{i}} m_{D_{j}} .
$$

In the seesaw approximation $\left(m_{D_{i}}<<M_{R}\right)$, the denominator of eq. (2.4) becomes only $M_{R}^{2}$. The five mass eigenstates of eq. (2.3) are denoted by $\widetilde{n}_{i}, i=1, \ldots, 5$, out of which $\tilde{n}_{1,2,3}$ are nothing but three light neutrinos.

Structure of eq. (2.4) tells us that only one neutrino is massive at the tree level with mass:

$$
m_{\nu_{3}}=\frac{\mu_{s}}{\left(M_{R}^{2}+\sum m_{D_{k}}^{2}\right)} \sum m_{D_{i}}^{2}
$$

The smallness of the neutrino mass is ascribed to the smallness of the $\mu_{s}$ parameter, rather than the largeness of the Majorana-type mass, $M_{R}$, as required for the standard seesaw mechanism [83]. With the choice of normal hierarchy in the light neutrino masses this tree level mass will attribute to the atmospheric scale $\sim 10^{-11} \mathrm{GeV}$. In the regime of seesaw approximation, for a typical Dirac mass $m_{D_{i}} \sim 10^{2} \mathrm{GeV}$ (assuming neutrino Yukawa couplings, $h_{\nu}^{i} \sim 10^{-1}$ ) and $\mathrm{TeV}$ scale $M_{R}$, the value of the parameter $\mu_{s}$ comes out to be $\sim 10^{-9} \mathrm{GeV}$. On the contrary, when $m_{D_{i}} \sim M_{R}$ (see eq. (2.4)) then the atmospheric neutrino scale $\left(\sim 10^{-11} \mathrm{GeV}\right)$ is determined by $\mu_{s}$ only.

The neutrino mass matrix shown in eq. (2.4) is diagonalizable using a $3 \times 3$ unitary matrix as

$$
U^{\operatorname{tr} T} M_{\text {tree }}^{\nu} U^{t r}=\operatorname{diag}\left(0,0, m_{\nu_{3}}\right) .
$$

The matrix $U^{t r}$ contains information about the tree level neutrino mixing angles. In order to satisfy the neutrino experimental data [1-3] one requires a second non-zero neutrino 
mass eigenvalue which can arise by including the one-loop corrections in the neutrino mass matrix [22].

In this model the doublet and singlet sneutrinos mix after the electroweak symmetry breaking. Thus the sneutrino mass squared matrix, $M_{\tilde{\nu}}^{2}$ is now a $10 \times 10$ matrix for MSISM, and assuming $C P$ conservation this matrix can be decomposed into two $5 \times 5$ block matrices corresponding to $C P$-even and $C P$-odd sneutrino fields. The sneutrino mass term in the Lagrangian, then looks like,

$$
\mathcal{L}_{\tilde{\nu}}=\frac{1}{2}\left(\phi^{R}, \phi^{I}\right)\left(\begin{array}{cc}
M_{+}^{2} & 0 \\
0 & M_{-}^{2}
\end{array}\right)\left(\begin{array}{c}
\phi^{R} \\
\phi^{I}
\end{array}\right),
$$

where, $\phi^{R}=\left(\widetilde{\nu}_{i}^{R}, \widetilde{\nu}^{c R}, \widetilde{S}^{R}\right), \phi^{I}=\left(\widetilde{\nu}_{i}^{I}, \widetilde{\nu}^{c I}, \widetilde{S}^{I}\right)$. The two mass squared matrices $M_{ \pm}^{2}$ are given by [31]

$$
M_{ \pm}^{2}=\left(\begin{array}{ccc}
\left(M_{\tilde{L}_{i}}^{2}+\frac{1}{2} M_{Z}^{2} \cos 2 \beta+m_{D_{i}}^{2}\right) \delta_{i j} \pm\left(A_{h_{\nu}}^{j} v_{u}-\mu m_{D_{j}} \cot \beta\right) & m_{D_{j}} M_{R} \\
\pm\left(A_{h_{\nu}}^{i} v_{u}-\mu m_{D_{i}} \cot \beta\right) & m_{\nu^{c}}^{2}+M_{R}^{2}+\sum m_{D_{k}}^{2} & \mu_{S} M_{R} \pm B_{M_{R}} \\
m_{D_{i}} M_{R} & \mu_{S} M_{R} \pm B_{M_{R}} & m_{S}^{2}+\mu_{S}^{2}+M_{R}^{2} \pm B_{\mu_{S}}
\end{array}\right) \text {, }
$$

where $M_{\widetilde{L}_{i}}^{2}$ denote soft supersymmetry breaking mass squared terms for $S U(2)_{L}$ doublet sleptons and $M_{Z}$ is the Z-boson mass. The ratio of the two Higgs VEVs is defined as $\tan \beta=\frac{v_{u}}{v_{d}}$, where $v_{d}$ is the vacuum expectation value of the down-type Higgs field $H_{d}$. The real symmetric mass matrix of eq. (2.7) can be diagonalized by a $10 \times 10$ orthogonal matrix as follows

$$
\mathbf{G} M_{\tilde{\nu}}^{2} \mathbf{G}^{T}=\operatorname{diag}\left(m_{\tilde{N}_{1}}^{2}, \ldots, m_{\tilde{N}_{10}}^{2}\right),
$$

with $m_{\widetilde{N}_{1}}^{2}<\ldots<m_{\widetilde{N}_{10}}^{2}$. Diagonalizing the $C P$-even and $C P$-odd mass matrices $M_{ \pm}^{2}$ separately by

$$
\mathbf{G}_{ \pm} M_{ \pm}^{2} \mathbf{G}_{ \pm}^{T}=\operatorname{diag}\left(m_{\widetilde{N}_{i \pm}}^{2}\right), i=1, \ldots, 5,
$$

where $\widetilde{N}_{i+}$ and $\widetilde{N}_{i-}$ denote the $i$-th $C P$-even and $C P$-odd sneutrino mass eigenstates, respectively, leads to a different parameterization which can be used in some cases. In this notation, for the set of chosen parameters (shown later) $\widetilde{N}_{1+}=\widetilde{N}_{2}$ and $\widetilde{N}_{1-}=\widetilde{N}_{1}$ and so on (see, eq. (2.9)).

\section{Decays of chargino and neutralino}

In this section we discuss the decays of charginos to charged leptons and singlet sneutrinos as well as the decays of the lighter neutralinos. We shall also show how these decays can lead to the final states, that we have proposed to study in this paper. Our choices of the four benchmark points for a detailed collider study will also be presented here.

\subsection{Chargino decay}

For the discussion of chargino decays we shall concentrate on a part of the parameter space where one of the singlet scalars of MSISM is the LSP. Hence this scalar singlet will appear 
at the end of the supersymmetric cascade decay chains. For the present discussion let us assume that the dominant decay mode of the lighter chargino is in the two body mode

$$
\tilde{\chi}_{1}^{ \pm} \rightarrow \widetilde{N}_{a}+l_{i}^{ \pm}, \quad a=1,2, \quad l_{i}=e, \mu, \tau,
$$

with $\widetilde{N}_{1}$ being the $C P$ conjugated state to $\widetilde{N}_{2}$. The relevant piece of the Lagrangian for the calculation of this decay width is

$$
L_{\ell \tilde{\chi}^{-} \widetilde{\nu}}=\overline{\widetilde{\chi}_{j}^{+}}\left(C_{i j a}^{L} P_{L}+C_{i j a}^{R} P_{R}\right) l_{i} \tilde{N}_{a}+\text { h.c. },
$$

where

$$
\begin{aligned}
C_{i j a}^{L} & =-\frac{1}{\sqrt{2}}\left[g \mathbf{V}_{j 1}^{*}\left(\mathbf{G}_{a i}-i \mathbf{G}_{a, i+5}\right)-h_{\nu}^{i} \mathbf{V}_{j 2}^{*}\left(\mathbf{G}_{a 4}-i \mathbf{G}_{a 9}\right)\right], \\
C_{i j a}^{R} & =\frac{1}{\sqrt{2}} Y_{\ell_{i}} \mathbf{U}_{j 2}\left(\mathbf{G}_{a i}-i \mathbf{G}_{a, i+5}\right) .
\end{aligned}
$$

The $Y_{\ell_{i}}$ s are the charged lepton Yukawa couplings and $\mathbf{U}, \mathbf{V}$ are two unitary $2 \times 2$ chargino mixing matrices such that $\mathbf{U}^{*} m_{2 \times 2} \mathbf{V}^{-1}=\operatorname{diag}\left(m_{\widetilde{\chi}_{1}^{ \pm}}, m_{\tilde{\chi}_{2}^{ \pm}}\right)$, where $m_{\tilde{\chi}_{1}^{ \pm}}, m_{\widetilde{\chi}_{2}^{ \pm}}$are the two physical chargino masses. The $2 \times 2$ mass matrix $m_{2 \times 2}$ in the charged gaugino-higgsino basis $\psi^{+^{T}}=-i \widetilde{\lambda}_{2}^{+}, \widetilde{H}_{u}^{+}, \psi^{-T}=-i \widetilde{\lambda}_{2}^{-}, \widetilde{H}_{d}^{-}$is given by

$$
m_{2 \times 2}=\left(\begin{array}{cc}
M_{2} & g v_{u} \\
g v_{d} & \mu
\end{array}\right) .
$$

Here $g$ is the $S U(2)_{L}$ gauge coupling.

The corresponding decay widths are given as

$$
\Gamma\left(\widetilde{\chi}_{1}^{ \pm} \rightarrow \widetilde{N}_{a}+l_{i}^{ \pm}\right)=\frac{\left(m_{\chi_{1}^{ \pm}}^{2}-m_{\widetilde{N}_{a}}^{2}\right)^{2}}{32 \pi m_{\chi_{1}^{ \pm}}^{3}}\left(\left|C_{i 1 a}^{L}\right|^{2}+\left|C_{i 1 a}^{R}\right|^{2}\right) .
$$

The members of $C P$ conjugated pair of sneutrinos being nearly mass degenerate $\left(m_{\widetilde{N}_{1}} \approx\right.$ $m_{\widetilde{N}_{2}}$ ) they are unlikely to be distinguished experimentally. Hence we sum over the $C P$-even and $C P$-odd sneutrino states of the $C P$ conjugated pair. Thus

$$
\Gamma\left(\widetilde{\chi}_{1}^{ \pm} \rightarrow \widetilde{N}_{1+2}+l_{i}^{ \pm}\right) \equiv \sum_{\alpha=1}^{2} \Gamma\left(\widetilde{\chi}_{1}^{ \pm} \rightarrow \widetilde{N}_{\alpha}+l_{i}^{ \pm}\right) .
$$

One can adjust the parameters $\mu_{S}$ and $B_{\mu_{S}}$ in such a way that the tree-level neutrino mass matrix contribution determines the atmospheric mass scale, while the one-loop corrections control the solar mass scale [22]. In such a situation it can be shown that in order to have small reactor neutrino mixing angle and maximal atmospheric neutrino mixing angle, the parameter $m_{D_{1}}$ has to be considerably smaller than other two Dirac masses and simultaneously, $m_{D_{2}} \sim m_{D_{3}}$. The solar neutrino mixing angle can be kept large by keeping the parameters $\delta_{i} \equiv A_{h_{\nu}}^{i} v_{u}-\mu m_{D_{i}} \cot \beta$ to be of the same order for all the three flavors, $i=e, \mu, \tau$. In this case, one can show that the decay width of the lighter chargino, 
$\Gamma\left(\widetilde{\chi}_{1}^{ \pm} \rightarrow \widetilde{N}_{1+2}+l_{i}^{ \pm}\right)$correlates with the corresponding parameter $m_{D_{i}}^{2}$. The atmospheric

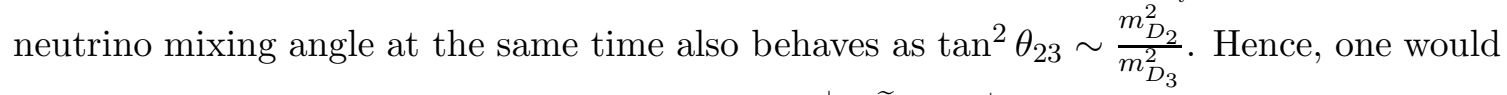
expect that the ratio of the branching ratios $\frac{\operatorname{Br}\left(\widetilde{\chi}_{1}^{ \pm} \rightarrow \widetilde{N}_{1+2}+\mu^{ \pm}\right)}{\operatorname{Br}\left(\widetilde{\chi}_{1}^{ \pm} \rightarrow \widetilde{N}_{1+2}+\tau^{ \pm}\right)}$must correlate with the ratio $\frac{m_{D_{2}}^{2}}{m_{D_{3}}^{2}}$. This has been shown in figure 1.

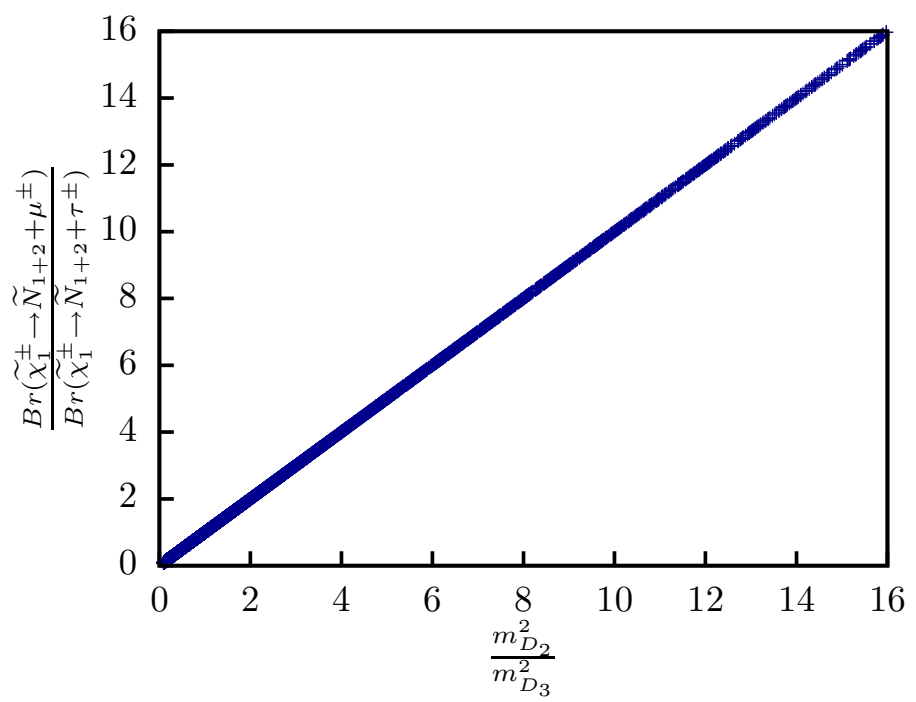

Figure 1: Correlation plot for the ratio of the branching ratios $\frac{B r\left(\widetilde{\chi}_{1}^{ \pm} \rightarrow \widetilde{N}_{1+2}+\mu^{ \pm}\right)}{\operatorname{Br}\left(\widetilde{\chi}_{1}^{ \pm} \rightarrow \widetilde{N}_{1+2}+\tau^{ \pm}\right)}$with $\frac{m_{D_{2}}^{2}}{m_{D_{3}}^{2}}$.

\subsection{Neutralino decay}

In our chosen benchmark points (defined later in this section) the lightest neutralino is the next-to-lightest supersymmetric particle (NLSP) and, decays dominantly through the two body decay channels $\tilde{\chi}_{1}^{0} \rightarrow \nu_{l_{i}}+\tilde{N}_{1,2}, l_{i}=e, \mu, \tau$. The relevant interaction term of the Lagrangian is:

$$
L_{\nu \widetilde{\chi}^{0} \widetilde{\nu}}=\overline{\widetilde{\chi}}_{j}^{0}\left(A_{m j b}^{L} P_{L}+A_{m j b}^{R} P_{R}\right) \nu_{m} \widetilde{N}_{b}+h . c .
$$

where

$$
\begin{aligned}
& A_{m j b}^{L}=\frac{g}{2}\left(\mathbf{N}_{j 2}^{*}-\tan \theta_{W} \mathbf{N}_{j 1}^{*}\right)\left(\mathbf{G}_{b i}-i \mathbf{G}_{b(i+5)}\right) U_{i m}^{t r}, \\
& A_{m j b}^{R}=-\frac{1}{\sqrt{2}} h_{\nu}^{i} U_{i m}^{t r} \mathbf{N}_{j 4}\left(\mathbf{G}_{b 4}-i \mathbf{G}_{b 9}\right) .
\end{aligned}
$$

Here $g$ is the $S U(2)_{L}$ gauge coupling, $\theta_{W}$ is the weak mixing angle, and $\mathbf{N}$ is the unitary $4 \mathrm{x} 4$ neutralino mixing matrix. Although the second lightest neutralino $\left(\widetilde{\chi}_{2}^{0}\right)$ decays mostly through the standard MSSM two-body charged lepton-slepton channel $\left(\widetilde{\chi}_{2}^{0} \rightarrow \widetilde{l}_{i}^{ \pm}+l_{i}^{\mp}\right)$, some of its branching fraction goes into the decay channels arising from the coupling given in eq. (3.7). Here, we have neglected the charged lepton flavor violating decay of $\widetilde{\chi}_{2}^{0}$. The decay width of a neutralino decaying into neutrino-sneutrino two-body mode is given as

$$
\Gamma\left(\widetilde{\chi}_{j}^{0} \rightarrow \widetilde{N}_{b}+\nu_{m}\right)=\frac{\left(m_{\chi_{j}^{0}}^{2}-m_{\widetilde{N}_{b}}^{2}\right)^{2}}{32 \pi m_{\chi_{j}^{0}}^{3}}\left(\left|A_{m j b}^{L}\right|^{2}+\left|A_{m j b}^{R}\right|^{2}\right)
$$




\subsection{Trilepton signal and the benchmark points}

In order to illustrate the trilepton signal we simulate $\widetilde{\chi}_{2}^{0} \widetilde{\chi}_{1}^{ \pm}$production followed by their two-body decays to produce $3 \ell+\mathbb{E}_{T}$ or $2 \ell+\tau-$ jet $+\mathbb{E}_{T}$ final states, where $\ell=e, \mu$.

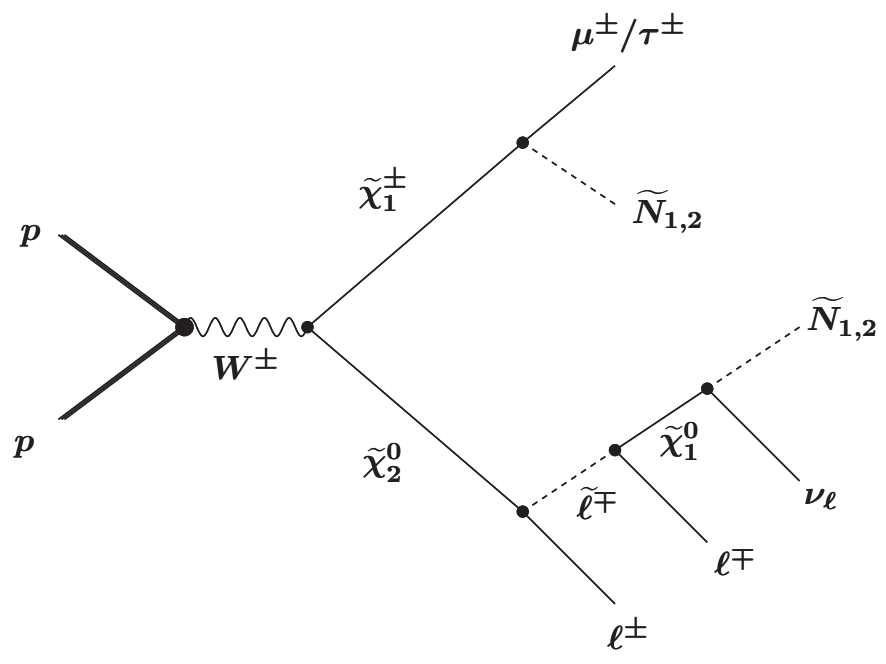

Figure 2: Feynman diagram for the process $p p \rightarrow 3 \ell+\mathscr{E}_{T}$ or $2 \ell+\tau-$ jet $+\mathbb{E}_{T}$.

As discussed above the production process and the decay cascades leading to these final states are as follows

$$
\begin{aligned}
& p p \rightarrow \widetilde{\chi}_{2}^{0}+\widetilde{\chi}_{1}^{ \pm}, \\
& \widetilde{\chi}_{1}^{ \pm} \rightarrow \widetilde{N}_{1,2}+\mu^{ \pm} / \tau^{ \pm}, \\
& \widetilde{\chi}_{2}^{0} \rightarrow \widetilde{\ell}^{ \pm}+\ell^{\mp}, \\
& \widetilde{\ell}^{ \pm} \rightarrow \ell^{ \pm}+\widetilde{\chi}_{1}^{0}, \\
& \widetilde{\chi}_{1}^{0} \rightarrow \nu_{l}+\widetilde{N}_{1,2} .
\end{aligned}
$$

The Feynman diagram for the above mentioned final states is shown in figure 2. In the presence of heavy squarks $(\sim 1 \mathrm{TeV})$, this is the leading process for the chosen signal.

Because of the presence of the massive singlet sneutrino LSPs, $\widetilde{N}_{1,2}$, (quasi-degenerate in masses), we have, for this model, a large amount of missing energy in the final states. In order to have an appreciable signal rate one must have significant production cross section of $\widetilde{\chi}_{2}^{0}-\widetilde{\chi}_{1}^{ \pm}$pair and large branching ratios for the above-mentioned decays. To achieve these we have chosen four benchmark points $(\mathrm{BPs})$ in the parameter space where the detailed collider simulation has been performed. We scanned the whole parameter space to check for charged lepton flavour violating (LFV) decay widths and we found points both above and below the experimental limits in different region of the parameter space. In all of the benchmark points, constraints from LFV decays [84] are satisfied as well as the atmospheric neutrino mixing is near maximal. The input parameters for different benchmark points are given in table 1. The choices of the parameters $m_{D_{i}}^{2}$ will be shown later.

The mass splittings between the second lightest neutralino, the charged sleptons and the lightest neutralino are maintained in a way, that the second lightest neutralino decays 


\begin{tabular}{|ccccc|}
\hline & BP1 & BP2 & BP3 & BP4 \\
\hline $\tan \beta$ & 5 & 10 & 20 & 10 \\
$\mu(\mathrm{GeV})$ & 330 & 240 & 280 & 350 \\
$M_{1}(\mathrm{GeV})$ & 170 & 195 & 160 & 240 \\
$M_{2}(\mathrm{GeV})$ & 220 & 340 & 240 & 290 \\
$M_{3}(\mathrm{GeV})$ & 1100 & 1100 & 1100 & 1100 \\
$M_{R}(\mathrm{GeV})$ & 145 & 160 & 140 & 150 \\
$\mu_{s} \times 10^{9}(\mathrm{GeV})$ & 7.80 & 7.81 & 7.75 & 7.76 \\
$m_{\nu^{c}}^{2}\left(\mathrm{GeV}^{2}\right)$ & 2500 & 3025 & 2500 & 3025 \\
$m_{S}^{2} \times 10^{-4}\left(\mathrm{GeV}^{2}\right)$ & 4.0 & 4.0 & 4.0 & 4.8 \\
$B_{M_{R}}\left(\mathrm{GeV}^{2}\right)$ & 2500 & 2500 & 3500 & 2500 \\
$B_{\mu_{S}}\left(\mathrm{GeV}^{2}\right)$ & 10 & 10 & 10 & 10 \\
$M_{\widetilde{L}_{i}}^{2} \times 10^{-5}\left(\mathrm{GeV}^{2}\right)$ & 5.63 & 5.63 & 5.63 & 5.63 \\
$M_{\widetilde{e}_{11}^{c}}^{2} \times 10^{-4}\left(\mathrm{GeV}^{2}\right)$ & 2.99 & 3.69 & 2.59 & 5.86 \\
$M_{\widetilde{e}_{22}^{c}}^{2} \times 10^{-4}\left(\mathrm{GeV}^{2}\right)$ & 2.99 & 3.69 & 2.59 & 5.86 \\
$M_{\widetilde{e}_{33}^{c}}^{2} \times 10^{-4}\left(\mathrm{GeV}^{2}\right)$ & 3.53 & 7.08 & 7.90 & 8.18 \\
\hline
\end{tabular}

Table 1: Values of the relevant input parameters for different benchmark points. The quantities $M_{\widetilde{e}_{i i}^{c}}^{2}$ represent soft squared masses for the right-handed charged sleptons. Other parameters are defined in the text.

only through charged lepton-slepton two body modes and the charged sleptons further decay into the lightest neutralino and charged lepton states. With these considerations we generated the sparticle spectrum using SuSpect (version 2.41) [85]. Masses of the neutrino and sneutrino states are computed using a self developed code in FORTRAN. Relevant mass spectra for these benchmark points are shown in table 2 .

The choice of model parameters for different benchmark points are chosen to yield statistically significant final states. As an illustrative example, production cross sections for the $\widetilde{\chi}_{2}^{0} \widetilde{\chi}_{1}^{ \pm}$pair with $7 \mathrm{TeV}$ center of mass energy at LHC are in the range of $200-300 \mathrm{fb}$ for the first and third benchmark points. For the fourth benchmark point with relatively heavy $\widetilde{\chi}_{2}^{0} \widetilde{\chi}_{1}^{ \pm}$pair (see table 2) the production cross section is reduced by a factor of $4(3)$ compared to the first(third) benchmark point. On the contrary, a higgsino like $\widetilde{\chi}_{2}^{0} \widetilde{\chi}_{1}^{ \pm}$pair (BP2) yield a similar production cross-section like BP4, in spite of having a lighter $\widetilde{\chi}_{2}^{0} \widetilde{\chi}_{1}^{ \pm}$ pair. Thus, the region of parameter space with higgsino like $\widetilde{\chi}_{2}^{0} \widetilde{\chi}_{1}^{ \pm}$pair is unfavorable for this analysis.

Note that hadronically quiet trilepton signal $\left(3 \ell+\mathbb{E}_{T}\right)$ will get very little contribution from squark-squark, squark-gluino and gluino-gluino pair production. On the other hand, when we have $2 \ell+\tau$-jet $+\mathbb{E}_{T}$ signal, then one should consider all other sources of dilepton +1 -jet $+\mathbb{E}_{T}$ events where one jet can be faked as a $\tau$-jet. For example, one can have a jet out of a squark decay $\left(\tilde{q} \rightarrow q^{\prime}+\widetilde{\chi}_{1}^{ \pm}\right)$from one side of the cascade. However, since in this model the squarks are much heavier $(\sim 1 \mathrm{TeV})$ and after incorporating the probability of any jet faking as a $\tau$-jet, the event rate comes out to be negligibly small compared to the one generated from chargino-neutralino production. Hence the main contribution to 
$2 \ell+\tau$-jet $+\mathbb{E}_{T}$ signal comes from $\widetilde{\chi}_{2}^{0} \widetilde{\chi}_{1}^{ \pm}$production only.

\section{Event generation and background analysis}

On the basis of the discussion presented in the previous section, let us now provide a detailed description of event generation and subsequently, the background analysis. The decay widths corresponding to the two-body modes shown in eq. (3.10) have been used to modify the branching fractions of the charginos and neutralinos obtained from Suspect. These input files are then fed to PYTHIA (version 6.409) [86] for event generation and showering. Initial and final state radiation, decay, hadronization, fragmentation and jet formation are implemented following the standard procedures in PYTHIA. Factorization and renormalization scales are set at $\sqrt{\widehat{s}}$ (i.e $\mu_{R}=\mu_{F}=\sqrt{\widehat{s}}$ ), where $\sqrt{\widehat{s}}$ is

\begin{tabular}{|ccccc|}
\hline & BP1 & BP2 & BP3 & BP4 \\
\hline$\widetilde{N}_{1+}$ & 153.27 & 169.18 & 147.96 & 159.76 \\
$\widetilde{N}_{2+}$ & 247.38 & 256.45 & 244.81 & 266.53 \\
$\widetilde{N}_{1-}$ & 153.27 & 169.18 & 147.96 & 159.76 \\
$\widetilde{N}_{2-}$ & 247.34 & 256.42 & 244.76 & 266.50 \\
$\widetilde{n}_{4}$ & 145.46 & 160.52 & 140.42 & 150.45 \\
$\widetilde{n}_{5}$ & 145.46 & 160.52 & 140.42 & 150.45 \\
$\widetilde{e}_{L}, \widetilde{\mu}_{L}$ & 751.28 & 751.37 & 751.39 & 751.37 \\
$\widetilde{e}_{R}, \widetilde{\mu}_{R}$ & 178.00 & 196.97 & 166.83 & 245.98 \\
$\widetilde{\tau}_{1}$ & 193.11 & 269.48 & 284.12 & 289.17 \\
$\widetilde{\tau}_{2}$ & 751.30 & 751.40 & 751.53 & 751.43 \\
$\widetilde{\chi}_{1}^{0}$ & 159.51 & 172.90 & 151.80 & 226.36 \\
$\widetilde{\chi}_{2}^{0}$ & 198.11 & 234.59 & 207.67 & 264.16 \\
$\widetilde{\chi}_{1}^{ \pm}$ & 192.79 & 215.47 & 203.72 & 255.99 \\
$\widetilde{\chi}_{2}^{ \pm}$ & 363.91 & 372.89 & 326.77 & 391.60 \\
\hline
\end{tabular}

Table 2: Relevant mass spectra obtained for four benchmark points with $\widetilde{n}_{1,2,3}=\nu_{1,2,3}$.

the parton level centre of mass energy. We have used the leading order CTEQ5L parton distribution functions $[87,88]$ for the colliding protons. Some of the background events are generated using ALPGEN (version 2.14) [89] with default factorization and renormalization scales. The jets are constructed using cone algorithm in PYCELL. Only those jets are constructed which have $p_{T}>20 \mathrm{GeV}$ and $|\eta|<2.5$. To simulate detector effects we have taken into account smearing of jet energies by a Gaussian probability density function of width [90] $\sigma(E) / E_{j}=\left(0.6 / \sqrt{E_{j}[G e V]}\right)+0.03$ where $E_{j}$ is the unsmeared jet energy.

In order to find three isolated leptons in the final states we impose following cuts and isolation criteria:

I. Leptonic events are selected only if $p_{T}^{\ell}>8 \mathrm{GeV}$ and $\left|\eta^{\ell}\right|<2.4$.

II. Lepton-lepton separation $\Delta R(\ell, \ell)$ set to be $>0.2$, where $\Delta R=\sqrt{(\Delta \eta)^{2}+(\Delta \phi)^{2}}$.

III. Lepton-jet separation $\Delta R(\ell, j)$ chosen to be $>0.5$.

IV. The sum of $E_{T}$ deposits of the hadrons which fall within a cone of $\Delta R \leq 0.2$ around a lepton, must be less than $10 \mathrm{GeV}$.

A $p_{T}$ cut of $10 \mathrm{GeV}$ and $17 \mathrm{GeV}$ [81] is applied on final state muons and electrons respectively, for the analysis at $7 \mathrm{TeV}$ and $14 \mathrm{TeV}$ center of mass energies at the LHC. The $\tau$-jets are counted with $p_{T} \geq 20 \mathrm{GeV}$ and $\left|\eta^{\tau}\right|<2.4$. The $\tau$ 's are then counted according to the visible energy bins. A $\tau$-jet is treated as tagged or untagged according to the efficiency $\left(\epsilon_{\tau}\right)$ of the most efficient algorithm given in [91]. In reference [91], $\tau$ identification efficiency 
obtained from actual collision data at $7 \mathrm{TeV}$ center of mass energy has also been quoted. The efficiencies obtained from Monte-Carlo simulation and from the data agrees very well. However, for higher luminosity with $14 \mathrm{TeV}$ center of mass energy, a lot of underlying events are expected to be there, which can perhaps bring down the detection efficiency. In this case also we have used the same efficiency as in $7 \mathrm{TeV}$ case hoping the experimentalists can maintain the efficiency as we have now. Unlike $\tau$, detection efficiencies of $e$ and $\mu$ are assumed to be $100 \%$.

We have analysed the SM backgrounds in some detail. The dominant background events arise from $t \bar{t}$ and $W Z$ production at the LHC. Apart from these, contributions from $Z Z, W W, Z b \bar{b}, W b \bar{b}, Z+$ jets, $W t, t b, W W W, W t \bar{t}$ events have also been studied at the leading order. We also studied $Q C D$ di-jet events. But after putting the cuts to reduce backgrounds as mentioned below we found no trilepton events for $1 \mathrm{fb}^{-1}$ integrated luminosity from these particular $Q C D$ events. We use ALPGEN for an estimation of $Z b \bar{b}$, $W b \bar{b}, W t, t b, Z+j e t s, W W W, W t \bar{t}$ backgrounds. We generate these events at the parton level using ALPGEN and fed those partonic events to PYTHIA for showering, hadronization, fragmentation, decay, etc. The other events are generated and analysed using PYTHIA. It should be mentioned that the importance of these processes have already been emphasized in the literature [74,92].

The trilepton signal in our model arising out of chargino-neutralino production is accompanied by large missing transverse energy $\left(\mathbb{E}_{T}\right)$, because of a pair of singlet sneutrino LSPs and a neutrino. As an example, the $\mathbb{E}_{T}$ spectrum of background events as well as the signal events $\left(3 \mu+\mathbb{E}_{T}\right)$ for the first benchmark point (BP1) are shown in figure 3 .

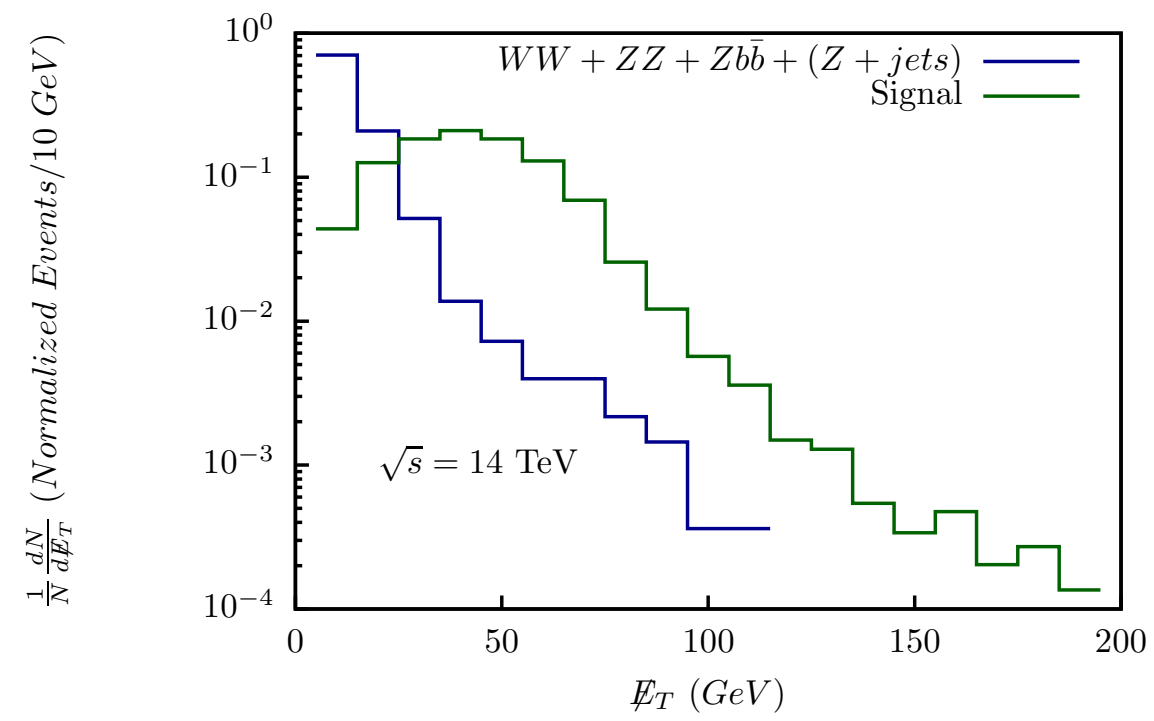

Figure 3: $\mathbb{E}_{T}$ plot for signal events $3 \mu+\not_{T}$ and summed up contribution coming from the $W W, Z Z, Z b \bar{b}, Z+1$ jet, $Z+2$ jets background for LHC at $14 \mathrm{TeV}$ center of mass energy with 1 $\mathrm{fb}^{-1}$ of integrated luminosity. The $\mathbb{E}_{T}$ bin size is chosen to be $10 \mathrm{GeV}$.

These distributions are obtained without applying any cuts to reduce background events. It is evident from the plot in figure 3 that a strong $\mathbb{E}_{T}$ cut will affect the signal 
cross-section very mildly, but it reduces significantly background events coming from some processes. Therefore, a cut $\mathbb{E}_{T}>25 \mathrm{GeV}$ is applied for background rejection. For some other channels; $t \bar{t}, W Z, W t \bar{t}, W W W$ the $\mathbb{E}_{T}$ distributions do not peak before $25 \mathrm{GeV}$ as shown in figure H. Hence, the above mentioned $\mathbb{E}_{T}$ cut does not seriously affect these background

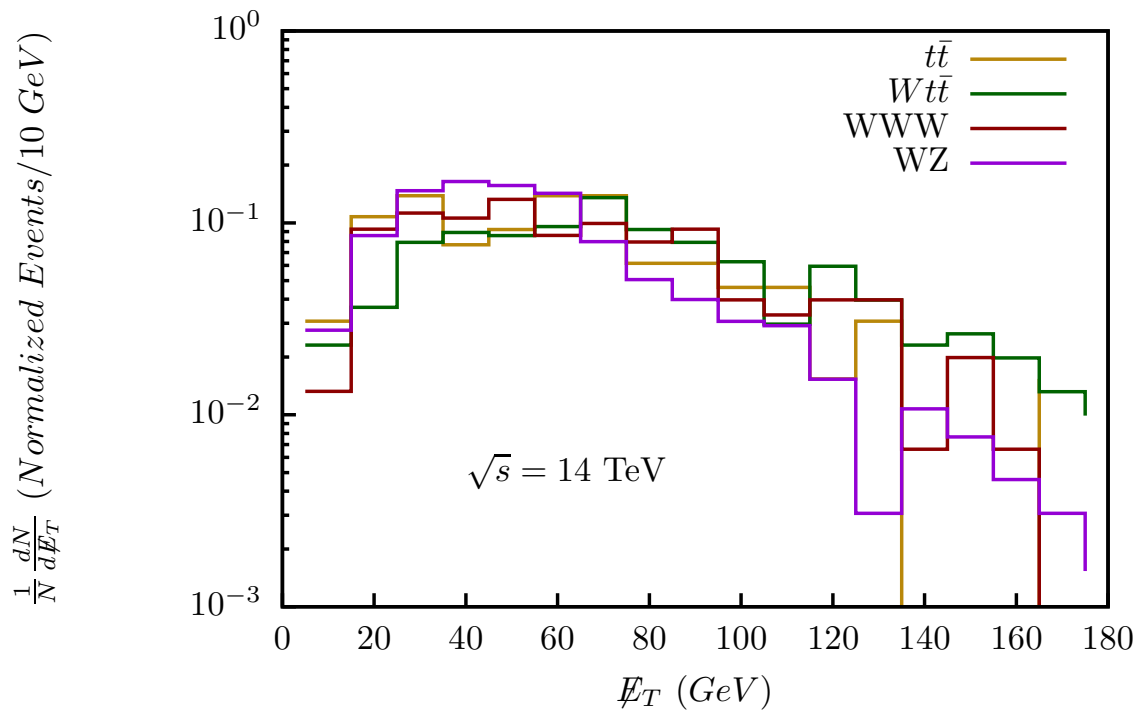

Figure 4: $\mathbb{E}_{T}$ plot for $3 \mu+\mathbb{E}_{T}$ events obtained from $t \bar{t}, W Z, W t \bar{t}, W W W$ backgrounds for LHC at $14 \mathrm{TeV}$ center of mass energy with $1 \mathrm{fb}^{-1}$ of integrated luminosity. The $\mathbb{E}_{T}$ bin size is chosen to be $10 \mathrm{GeV}$.

events. To reduce these events we have further applied two more cuts. An invariant mass cut on the opposite sign dilepton pair, $80 \mathrm{GeV}>M_{i n v}^{\ell \ell}>100 \mathrm{GeV}$ removes backgrounds coming from $Z$-bosons. To manifest this idea we show invariant mass distribution in figure 5 constructed from opposite sign muon pairs for signal events and $W Z$ background events.

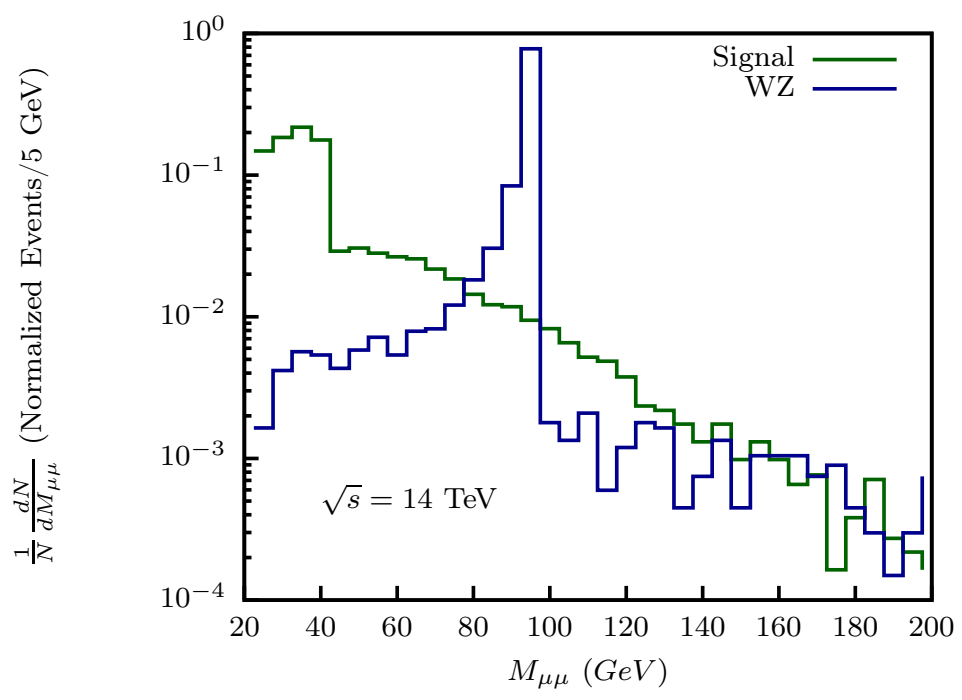

Figure 5: $M_{\mu \mu}$ plot for signal events and $W Z$ background for LHC at $14 \mathrm{TeV}$ center of mass energy. The $M_{\mu \mu}$ bin size is chosen to be $5 \mathrm{GeV}$. 
On the other hand, rejection of tagged $b$-jet events significantly reduces backgrounds coming from $t \bar{t}$ events. A jet (with $|\eta|<2.5$ ) is reconstructed as a $b$-jet if the $\Delta R$ separation between the jet and the $b$-quark (with $p_{T}>5 \mathrm{GeV}$ ) is less than 0.2 . The b-jet identification efficiency is taken to be $50 \%$.

In order to perform the collider analysis we have randomly generated $m_{D_{i}}^{2}$ and $\delta_{i}^{2}$ within certain range: $\left(\sum_{i} m_{D_{i}}^{2}\right)^{1 / 2} \in 10^{[-4,2.6]}$ and $\left(\sum_{i} \delta_{i}^{2}\right)^{1 / 4} \in 10^{[-4,3]}[22]$. Moreover, we also consider $\left(\sum m_{D_{i}}^{2}\right)^{1 / 2}>\left(\sum_{i} \delta_{i}^{2}\right)^{1 / 4}$, such that Dirac neutrino masses give the dominant contribution to the chargino decay [22]. Around each of the four benchmark points we select a set of six to seven points of these randomly generated parameters. These points will be useful for the correlation study discussed later in section 5 . Remember that these parameters control the neutrino masses and the mixing angles and our choices of benchmark points are such that the atmospheric neutrino mass scale is determined by the tree level neutrino mass matrix contribution. Before showering in PYTHIA, as mentioned earlier, the ratio $\frac{B r\left(\widetilde{\chi}_{1}^{ \pm} \rightarrow \widetilde{N}_{1+2}+\mu^{ \pm}\right)}{\operatorname{Br}\left(\widetilde{\chi}_{1}^{ \pm} \rightarrow \widetilde{N}_{1+2}+\tau^{ \pm}\right)}$shows a very nice sharp correlation when plotted against $\frac{m_{D_{2}}^{2}}{m_{D_{3}}^{2}}$ which is a measure of $\tan ^{2} \theta_{23}$. We have done the showering for four benchmark points introduced in table 1 and table 2 to look for the ratio $\frac{\sigma\left(p p \rightarrow \mu \sum \ell \ell+E_{T}\right)}{\sigma\left(p p \rightarrow \tau \sum \ell \ell+E_{T}\right)}$ with $\ell=e, \mu$. Since one $\mu$ and one $\tau$ in these final states always come from the decay of $\widetilde{\chi}_{1}^{ \pm}$, we would expect that this ratio will also go as $\sim \tan ^{2} \theta_{23}$. Hence, by measuring this ratio from the trilepton signals one can obtain information about the atmospheric neutrino mixing angle at the LHC. On the other hand, a precise measurement of the atmospheric neutrino mixing angle at the oscillation experiments can be used to predict the allowed range of the above ratio at the LHC. In the following section we give a quantitative estimate of this ratio for our choices of benchmark points (along with randomly selected values of $m_{D_{i}}^{2}$ ) and show that for each of these points the various signal events included in the calculation of this ratio can be statistically significant.

\section{Results}

In order to study the correlation between the atmospheric neutrino mixing angle $\left(\theta_{23}\right)$ and the final states with trilepton $+\mathbb{E}_{T}$ at the LHC, we look at the ratio of cross sections $\frac{\sigma\left(p p \rightarrow \mu \sum \ell \ell+\mathbb{E}_{T}\right)}{\sigma\left(p p \rightarrow \tau \sum \ell \ell+E_{T}\right)}, \ell=e, \mu$. As mentioned in the introduction, in the denominator the $\tau$ must always come from the decay of $\tilde{\chi}_{1}^{ \pm}$because we are considering final states with only one $\tau$-jet and neglecting lepton flavor violating decays of $\tilde{\chi}_{2}^{0}$ and $\tilde{\ell}^{ \pm}$. For the same reason, in the numerator one $\mu$ must always also come from the decay of $\tilde{\chi}_{1}^{ \pm}$. Hence, naively we would expect that this ratio of cross sections will also show nice correlation with the atmospheric neutrino mixing angle $\theta_{23}$.

After applying different cuts to reduce backgrounds and taking into account the $\tau$ tagging efficiency, we find that the ratio of trilepton signal cross section again shows a nice correlation with the atmospheric neutrino mixing angle $\tan ^{2} \theta_{23}$. However, in this case the numbers change from the ratio of branching ratios, discussed earlier and the straight lines obtained are steeper than the one shown in figure 1. This happens because in our simulation we take the detection efficiency of $\mu$ to be $100 \%$ as opposed to the $\tau$ detection efficiency, 
which is smaller [81]. Since the branching fractions of $\tau$ events are in the denominator of the ratio, the numbers naturally go up.

The cross-sections and the corresponding statistical significance $\left(\frac{S_{x}}{\sqrt{B_{x}+S_{x}}}\right.$ with $x=$ $e, \mu, \tau)$ obtained from our simulation for LHC are shown in this section. Here $S_{x}$ is defined as the number of $x \sum \ell \ell$ signal events and $B_{x}$ is defined as the number of corresponding background events. In more simple form significance for the $\mu \sum \ell \ell+\mathbb{E}_{T}$ channel is defined as $\frac{S_{\mu e e}+S_{\mu \mu \mu}}{\sqrt{S_{\mu e e}+S_{\mu \mu \mu}+B_{\mu e e}+B_{\mu \mu \mu}}}$. In a similar fashion significance for the $\tau \sum \ell \ell+\mathbb{E}_{T}$ channel can be obtained.

We quote the results below for an integrated luminosity of $25 \mathrm{fb}^{-1}$ for the LHC with $7 \mathrm{TeV}$ and $14 \mathrm{TeV}$ center-of-mass energies. The results are obtained with the cuts mentioned in section 4 . Throughout this analysis we have used leading order cross sections for the signals as well as all the backgrounds at the LHC. However, if next-to-leading order (NLO) corrections are included the statistical significance will not change much. For example, if NLO corrections are included the signal cross section at $14 \mathrm{TeV}$ LHC is expected to increase by 1.25 to 1.35 [93]. As discussed above, a large contribution to the background comes from the $t \bar{t}$ events. The NLO cross section for $t \bar{t}$ production at $14 \mathrm{TeV} \mathrm{LHC}$ is about $800 \mathrm{pb}[94,95]$ which is about a factor of two larger than the leading order cross section that we have used in our analysis. Thus taking into account the NLO contribution of all the major background events along with the signal event, the significance $S_{x} / \sqrt{B_{x}+S_{x}}$ estimated for our signal, will not change much and remains conservative in comparison to the uncertainties in the production cross sections.

Values of the randomly generated parameters $m_{D_{i}}^{2}$, for four chosen benchmark points are presented in table 3. For the numerical analysis we choose to vary $m_{D_{1}}^{2}$ in the range of $10^{-4}-10^{-2} \mathrm{GeV}^{2}$, whereas $m_{D_{2,3}}^{2}$ are varied within $10^{-2}$ to $10^{2} \mathrm{GeV}^{2}$. The $\delta_{i}^{2}$ are also varied accordingly, but keeping the constraints $\left(\sum m_{D_{i}}^{2}\right)^{1 / 2}>\left(\sum \delta_{i}^{2}\right)^{1 / 4}$. The scale of $m_{D_{i}}^{2}$ has a strong influence on the decay processes $\widetilde{\chi}_{1}^{ \pm} \rightarrow \widetilde{N}_{1+2}+\mu^{ \pm} / \tau^{ \pm}$and $\widetilde{\chi}_{j}^{0} \rightarrow \widetilde{N}_{b}+\nu_{m}$. In order to achieve a statistically significant trilepton final state originating from $\widetilde{\chi}_{2}^{0} \widetilde{\chi}_{1}^{ \pm}$pair, we would like to have $\operatorname{Br}\left(\widetilde{\chi}_{1}^{ \pm} \rightarrow \widetilde{N}_{1+2}+\mu^{ \pm} / \tau^{ \pm}\right)$to be large and $\operatorname{Br}\left(\widetilde{\chi}_{2}^{0} \rightarrow \widetilde{N}_{b}+\nu_{m}\right)$ to be small, simultaneously. However, in the limit $m_{D_{i}} \sim M_{R} \sim \mathcal{O}\left(10^{2} \mathrm{GeV}\right)$, the neutrino Yukawa couplings $h_{\nu}^{i}$ are $\sim \mathcal{O}(1)$. Then as can be seen from eqs. (3.5) and (3.9) both of these decay widths are large and consequently, yields a smaller branching ratio for $\widetilde{\chi}_{2}^{0} \rightarrow \widetilde{\ell}^{ \pm}+\ell^{\mp}$. We observe that in this case it is rather difficult to achieve a statistically significant final state particularly for the $\tau \sum \ell \ell+\mathbb{E}_{T}$ mode.

In the trilepton signals studied in this work, one lepton comes from the lighter chargino $\left(\widetilde{\chi}_{1}^{ \pm}\right)$decay and the other two same flavour opposite sign leptons come from the second lightest neutralino $\left(\widetilde{\chi}_{2}^{0}\right)$ decay. Since the probability of getting electrons from the chargino decay is suppressed compared to muons or taus,

\begin{tabular}{|ccccc|}
\hline$m_{D_{i}}^{2}\left(\mathrm{GeV}^{2}\right)$ & BP1 & BP2 & BP3 & BP4 \\
\hline$m_{D_{1}}^{2} \times 10^{4}$ & 2.12 & 3.30 & 1.58 & 4.24 \\
$m_{D_{2}}^{2}$ & 71.80 & 62.96 & 68.50 & 80.33 \\
$m_{D_{3}}^{2}$ & 62.45 & 54.87 & 66.63 & 86.00 \\
\hline
\end{tabular}

Table 3: Randomly generated values of $m_{D_{i}}^{2}$ corresponding to the four benchmark points as indicated in section 3 .

events with odd number of electrons (eee and $e \mu \mu)$ should have smaller cross-sections 
compared to others, which is clearly reflected in the signal cross-sections. This feature is intrinsically related with the small but non-zero reactor neutrino angle [96], which will be discussed again later. In table 4 and 6 chosen trilepton $+\mathbb{E}_{T}$ cross sections are shown along with the total standard model background cross section for the LHC at center-of-mass energy, $\sqrt{s}=7$ and $14 \mathrm{TeV}$, respectively. The corresponding statistical significance of the signals are shown respectively in table 5 and table 7 . We can see from table 5 that, at the LHC even with $\sqrt{s}=7 \mathrm{TeV}$, the lowest signal significance for $\tau \sum \ell \ell+\mathbb{E}_{T}$ final state, that we have obtained, is greater than $3 \sigma$ for an integrated luminosity of $25 \mathrm{fb}^{-1}$. Hence, the trilepton $+\mathscr{E}_{T}$ data for $25 \mathrm{fb}^{-1}$ integrated luminosity at $7 \mathrm{TeV}$ LHC should be able to constrain the theoretical parameter space of this model. These numbers (significance) are much higher for LHC with $\sqrt{s}=14 \mathrm{TeV}$ and are shown in table 7 . It is once again evident from these tables that a higgsino like $\widetilde{\chi}_{2}^{0} \widetilde{\chi}_{1}^{ \pm}$pair (BP2) yields statistically less significant specific trilepton final state. In other words for such benchmark points, the significance of the final state trilepton signal is less promising. This situation is comparable to a heavy gaugino like $\widetilde{\chi}_{2}^{0} \widetilde{\chi}_{1}^{ \pm}$pair as represented by $\mathrm{BP} 4$.

\begin{tabular}{|c|c|c|c|c|c|}
\hline $\begin{array}{c}\text { Tri-lepton } \\
\text { events }\end{array}$ & & $\begin{array}{l}\text { signal } \\
\sigma(\mathrm{fb})\end{array}$ & & & $\begin{array}{c}\text { Background } \\
\sigma(\mathrm{fb})\end{array}$ \\
\hline & $\mathrm{BP} 1$ & BP2 & BP3 & $\mathrm{BP} 4$ & \\
\hline eee & 0.37 & 0.31 & 0.50 & 0.23 & 8.73 \\
\hline$е e \mu$ & 9.47 & 5.37 & 8.30 & 3.12 & 18.91 \\
\hline$e \mu \mu$ & 1.08 & 0.49 & 1.26 & 0.66 & 21.15 \\
\hline$\mu \mu \mu$ & 24.13 & 8.21 & 18.85 & 8.51 & 23.84 \\
\hline$e e \tau$ & 2.93 & 2.14 & 2.86 & 1.40 & 4.60 \\
\hline$\mu \mu \tau$ & 7.17 & 3.39 & 6.99 & 4.04 & 13.18 \\
\hline
\end{tabular}

Table 4: Cross-section for different trilepton channels are shown here for four different benchmark points along with their total SM background contribution for LHC with $\sqrt{s}=7 \mathrm{TeV}$. Corresponding input parameters and mass spectrum are given in table 1, table 3 and table 2 , respectively. .

\begin{tabular}{|ccccc|}
\hline $\begin{array}{c}\text { Tri-lepton } \\
\text { events }\end{array}$ & & $\begin{array}{c}\text { Significance } \\
\frac{S_{x \sum \ell \ell}}{\sqrt{B_{x \sum \ell \ell}+S_{x \sum \ell \ell}}}\end{array}$ & & \\
\hline & $\mathrm{BP} 1$ & $\mathrm{BP} 2$ & $\mathrm{BP} 3$ & $\mathrm{BP} 4$ \\
\hline$\mu e e+\mu \mu \mu$ & 19.23 & 9.05 & 16.24 & 7.89 \\
$\tau e e+\tau \mu \mu$ & 9.57 & 5.72 & 9.37 & 5.65 \\
\hline
\end{tabular}

Table 5: Statistical significance of the studied trilepton signals with integrated luminosity $25 \mathrm{fb}^{-1}$ at the LHC for $\sqrt{s}=7 \mathrm{TeV}$ for different benchmark points.

We present the correlation plots, obtained with different randomly generated values of $m_{D_{i}}^{2}$ and $\delta_{i}^{2}$ around each of the four benchmark points. These are shown in figure 6 and figure 7 for the LHC with $\sqrt{s}=7 \mathrm{TeV}$ and $14 \mathrm{TeV}$, respectively. We present these correlations with best fit lines. It can be seen from these figures that the $3 \sigma$ allowed value of $\tan ^{2} \theta_{23}$ [1] from atmospheric neutrino oscillation experiments predict a value of the 


\begin{tabular}{|c|c|c|c|c|c|}
\hline $\begin{array}{c}\text { Tri-lepton } \\
\text { events }\end{array}$ & & $\begin{array}{l}\text { signal } \\
\sigma(\mathrm{fb})\end{array}$ & & & $\begin{array}{c}\text { Background } \\
\sigma(\mathrm{fb})\end{array}$ \\
\hline & BP1 & BP2 & BP3 & BP4 & \\
\hline eee & 1.29 & 0.91 & 1.56 & 0.75 & 24.92 \\
\hline$е e \mu$ & 27.63 & 15.65 & 23.50 & 10.48 & 91.64 \\
\hline$e \mu \mu$ & 3.00 & 1.66 & 3.59 & 2.14 & 117.97 \\
\hline$\mu \mu \mu$ & 65.42 & 24.32 & 53.10 & 26.47 & 85.94 \\
\hline$e e \tau$ & 8.82 & 6.48 & 9.11 & 4.76 & 29.16 \\
\hline$\mu \mu \tau$ & 20.11 & 9.80 & 18.46 & 12.48 & 56.29 \\
\hline
\end{tabular}

Table 6: Cross-section for different trilepton channels are shown here for four different benchmark points along with their total SM background contribution for LHC with $\sqrt{s}=14 \mathrm{TeV}$. Corresponding input parameters and mass spectrum are given in table 1, table 3 and table 2, respectively.

\begin{tabular}{|ccccc|}
\hline $\begin{array}{l}\text { Tri-lepton } \\
\text { events }\end{array}$ & & $\begin{array}{c}\text { Significance } \\
\frac{S_{x \sum \ell \ell}}{\sqrt{B_{x \sum \ell \ell}+S_{x \sum \ell \ell}}}\end{array}$ & & \\
\hline & $\mathrm{BP} 1$ & $\mathrm{BP} 2$ & $\mathrm{BP} 3$ & $\mathrm{BP} 4$ \\
\hline$\mu e e+\mu \mu \mu$ & 28.28 & 13.55 & 24.02 & 12.61 \\
$\tau e e+\tau \mu \mu$ & 13.53 & 8.07 & 12.97 & 8.50 \\
\hline
\end{tabular}

Table 7: Statistical significance of the studied trilepton signals with integrated luminosity $25 \mathrm{fb}^{-1}$ at the LHC for $\sqrt{s}=14 \mathrm{TeV}$ for different benchmark points.

ratio of cross sections $\frac{\sigma\left(p p \rightarrow \mu \sum \ell \ell+\mathbb{E}_{T}\right)}{\sigma\left(p p \rightarrow \tau \sum \ell \ell+\mathbb{E}_{T}\right)},(\ell=e, \mu)$ to be approximately in the range $1.0-6$. These predictions can be verified at the LHC or the measured value of this ratio can give an alternative estimate of $\tan ^{2} \theta_{23}$. On the other hand, if this ratio comes out to be very much different from the ones predicted here then one can perhaps conclude that MSISM is not the correct model for explaining neutrino masses and mixing.

Nevertheless, as we can see, from the correlation plots, that there is a different linear relationship for each different kind of benchmark points. In general then, from neutrino oscillation data we cannot give a unique prediction for the ratio of the cross-sections that can be verified at the LHC and help us in constraining the model parameters. In other words, measuring the cross section ratio at the LHC would not allow a prediction of $\theta_{23}$ that could be tested against oscillation results. This means that we need other measurements at the LHC to allow such predictions. As an example, to distinguish among the four benchmark points we plot the ratio, $m_{\widetilde{\chi}_{1}^{ \pm}} / m_{\widetilde{N}_{1,2}}$ with the ratio of cross-sections of $\mu$ and $\tau$ channels which gives four separate parallel lines for the four benchmark points (figure 8). One can see from figure 8 that the ratio $\left(m_{\widetilde{\chi}_{1}^{ \pm}} / m_{\widetilde{N}_{1,2}}\right)$ increases as the slope of the straight lines in the correlation plot corresponding to different benchmark points decreases. This pattern can easily be understood. Increase in the mass ratio indicates greater splitting between the chargino and sneutrino masses. As the splitting increases, the leptons coming from this chargino decay become more energetic (eq.(3.10)). This affects the $\tau$ count in the final state more than the $\mu$ count as the detection efficiency for the taus increases with the 


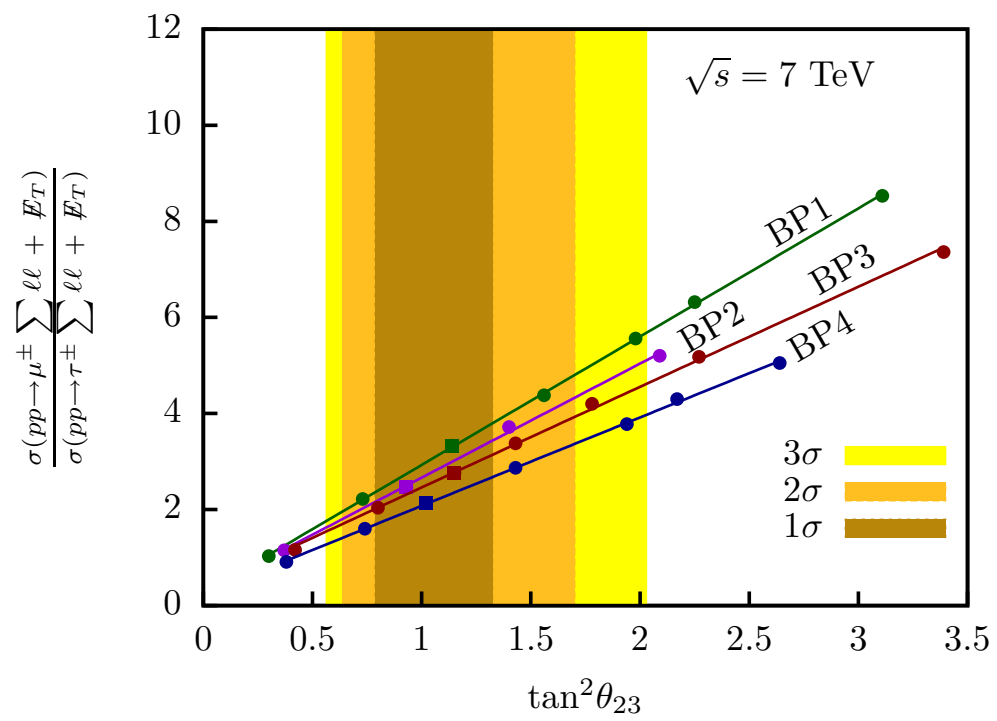

Figure 6: Correlation plot $\left(\frac{\sigma\left(p p \rightarrow \mu^{ \pm} \sum \ell \ell+E_{T}\right)}{\sigma\left(p p \rightarrow \tau^{ \pm} \sum \ell \ell+E_{T}\right)}\right.$ vs $\tan ^{2} \theta_{23}$, with $\left.\ell=e, \mu\right)$ obtained for LHC at $\sqrt{s}=7 \mathrm{TeV}$. Three differently coloured vertical strips correspond to $1 \sigma, 2 \sigma$ and $3 \sigma$ allowed region for $\tan ^{2} \theta_{23}$, respectively. The benchmark points as given in table 1, table 2 and table 3 are represented by coloured $\mathbf{\square}$. Other points, represented by coloured $\bullet$, are obtained with randomly generated $m_{D_{i}}^{2}$ and $\delta_{i}^{2}$ values.

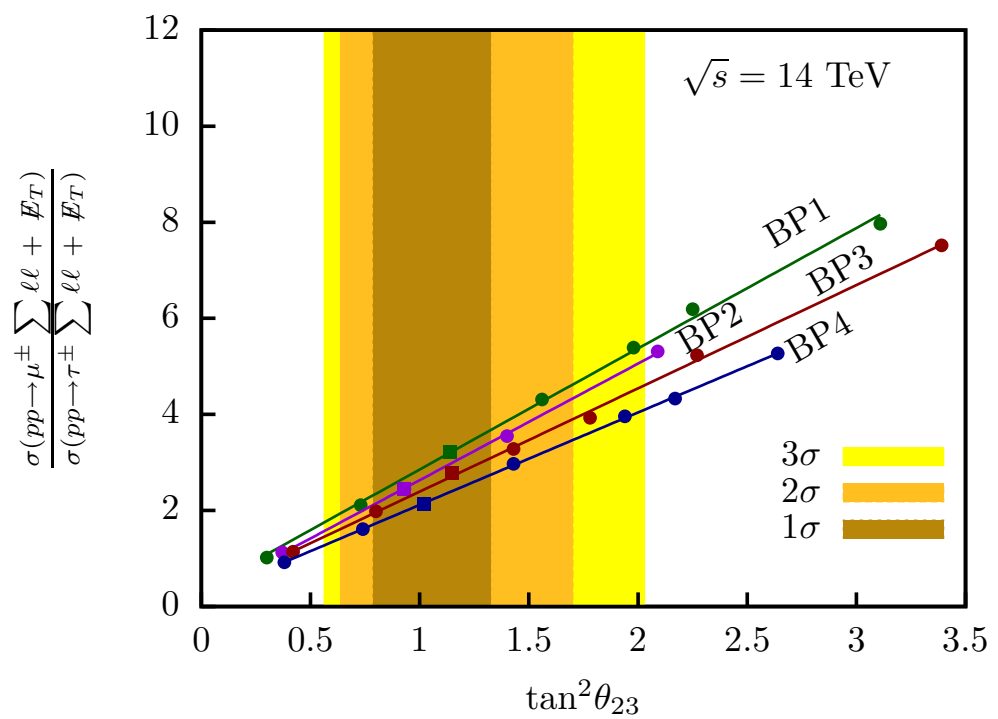

Figure 7: Correlation plot $\left(\frac{\sigma\left(p p \rightarrow \mu^{ \pm} \sum \ell \ell+E_{T}\right)}{\sigma\left(p p \rightarrow \tau^{ \pm} \sum \ell \ell+E_{T}\right)}\right.$ vs $\tan ^{2} \theta_{23}$, with $\left.\ell=e, \mu\right)$ obtained for LHC at $\sqrt{s}=14 \mathrm{TeV}$. Other specifications are the same as in figure 6 .

increase of visible energy of $\tau$ decay products [91]. Hence more $\tau$ events are expected in the final state for those benchmark points which has greater lighter chargino - LSP mass ratio for a given set of $m_{D_{i}}$ 's. With the increase of $\tau$ events the ratio of the cross-sections plotted in the correlation plots decreases and as a consequence gives smaller slope compared to the previous benchmark point. Now it is clearly understood that if we can determine the 


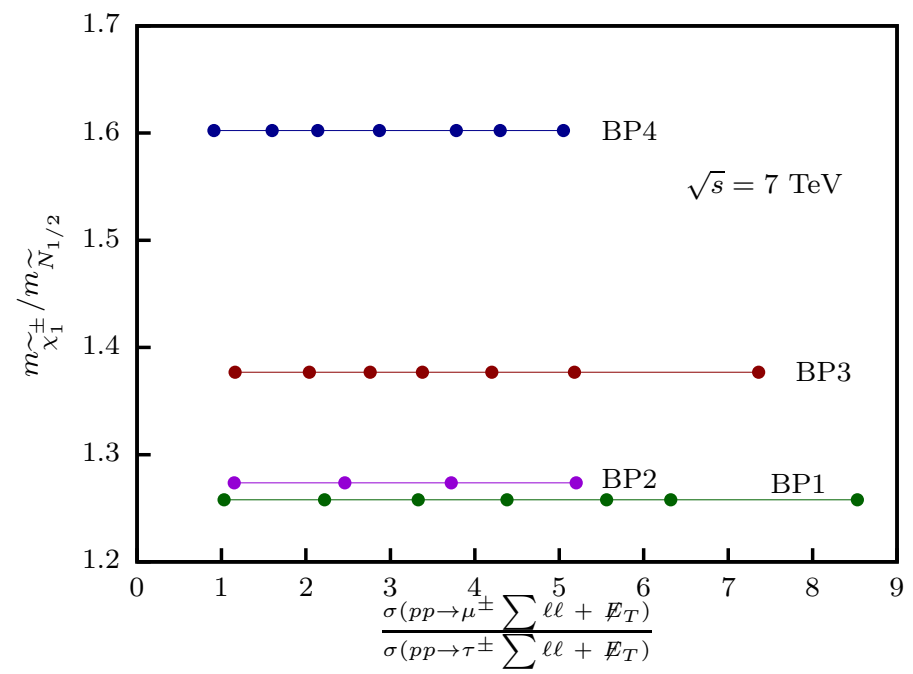

Figure 8: $m_{\widetilde{\chi}_{1}^{ \pm}} / m_{\widetilde{N}_{1,2}}$ plotted against $\frac{\sigma\left(p p \rightarrow \mu^{ \pm} \sum \ell \ell+E_{T}\right)}{\sigma\left(p p \rightarrow \tau^{ \pm} \sum \ell \ell+E_{T}\right)}$ for $\sqrt{s}=7 \mathrm{TeV}$. The mass ratios increase as the slopes of the straight lines in figure 6 corresponding to the four benchmark points decrease.

lighter chargino-LSP mass ratio, we can distinguish among the four benchmark points.

Mass determination techniques in the context of LHC have been studied extensively. Transverse mass variable $\left(m_{T_{2}}\right)[97,98]$ is very useful for this purpose. $m_{T_{2}}$ has also been generalized for the cases where the parent and daughter particles in the two decay chains are not identical $[99,100]$. Moreover, final state with more than two invisible particles has also been addressed in ref. [101]. In our case, we observe the following:

- The lightest neutralino $\widetilde{\chi}_{1}^{0}$ is also invisible, as mentioned earlier in the text.

- One lepton is produced from one side of the cascade and remaining two leptons from the other side of the cascade (figure 2).

- $\widetilde{\chi}_{1}^{ \pm}$and $\widetilde{\chi}_{2}^{0}$ are not mass degenerate but the difference is quite small in the context of mass measurement.

So, we see that daughters of different masses are produced here from nearly identical parents. A mass determination technique similar to refs. [99-101] can be applied here too to determine the masses of the lighter chargino and the sneutrino LSP. However, a detailed analysis in this direction is beyond the scope of the present paper. Thus we see that measuring the mass ratio $\left(m_{\widetilde{\chi}_{1}^{ \pm}} / m_{\widetilde{N}_{1,2}}\right)$, along with the ratio of the trilepton cross-section, can help us pick the correct benchmark point and hence predict the correct value of $\theta_{23}$ that could be tested against the oscillation results. On the other hand, a precise determination of $\tan ^{2} \theta_{23}$ from oscillation experiments as well as a measurement of the cross section ratio at the LHC can give a unique prediction of the mass spectrum of the model, that can be verified by mass measurements at the LHC.

In support of our explanation for obtaining different slopes, we present the following analysis. Since this difference among the four benchmark points appears because of taking 
different $\tau$ identification efficiencies for different energy range and for taking separate $p_{T}$ cuts for $\mu$ 's and $\tau$ 's, we can remove this by the following strategy:

- A $p_{T}$ cut of $20 \mathrm{GeV}$ taken for both $\mu$ and $\tau$.

- A uniform $\tau$ identification efficiency of $50 \%$ applied over the whole energy range.

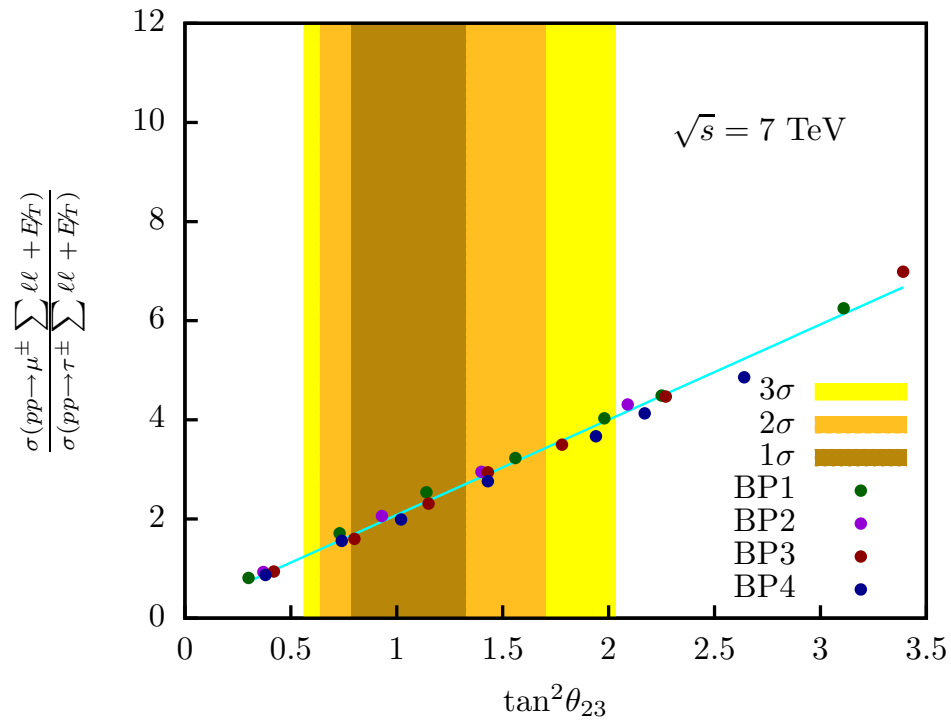

Figure 9: Correlation plot $\left(\frac{\sigma\left(p p \rightarrow \mu^{ \pm} \sum \ell \ell+E_{T}\right)}{\sigma\left(p p \rightarrow \tau^{ \pm} \sum \ell \ell+E_{T}\right)}\right.$ vs $\left.\tan ^{2} \theta_{23}\right)$ obtained for $7 \mathrm{TeV}$ center of mass energy under the assumption of uniform $\tau$ identification efficiency $\left(\epsilon_{\tau} \sim 0.5\right)$ and same $p_{T}$ cut for both $\mu$ and $\tau$.

We have presented the result in fig.9. This shows the correlation plot for $7 \mathrm{TeV}$ center of mass energy under the above mentioned conditions. All the benchmark points now lie almost on one straight line. A few points still looks a little bit scattered because of the different isolation criteria used for $\mu$ and $\tau$.

Finally, in table 8 we show the ratios $\frac{\sigma\left(p p \rightarrow e \mu \mu+\mathbb{E}_{T}\right)}{\sigma\left(p p \rightarrow 3 \ell+\mathbb{E}_{T}\right)}$ and $\frac{\sigma\left(p p \rightarrow e e e+\mathbb{E}_{T}\right)}{\sigma\left(p p \rightarrow 3 \ell+\mathbb{E}_{T}\right)}$ for the four benchmark points. The smallness of these ratios is also a distinct feature of this model and arises due to the smallness of the neutrino reactor angle imposed by neutrino data [96]. In the usual MSSM scenario these ratios are expected to be much higher as there is no suppression of charginos decaying into electrons as we have in this model.

A discussion of these specific trilepton signals remains incomplete without a note on the Tevatron analysis of the considered model. For the four chosen benchmark points we observed no points with significance $\geq 3 \sigma$ for the $\tau \sum \ell \ell+\mathbb{E}_{T}$ final state and simultaneously consistent with the atmospheric neutrino mixing at the $3 \sigma$ limit. This is a well expected result considering that the Tevatron center-of-mass energy is $1.96 \mathrm{TeV}$ with $12 \mathrm{fb}^{-1}$ of integrated luminosity [102]. For example, the statistical significance for $\tau \sum \ell \ell$ mode for BP1 with $m_{D_{i}}^{2}$ s given in table 3 is computed to be 1.64 . 


\begin{tabular}{|cccc|}
\hline & & $\frac{\sigma\left(p p \rightarrow e \mu \mu+E_{T}\right)}{\sigma\left(p p \rightarrow 3 \ell+E_{T}\right)} \times 10^{2}$ & $\frac{\sigma\left(p p \rightarrow e e e+E_{T}\right)}{\sigma\left(p p \rightarrow 3 \ell+E_{T}\right)} \times 10^{2}$ \\
\hline & $\sqrt{s}=7 \mathrm{TeV}$ & 3.09 & 1.06 \\
BP1 & $\sqrt{s}=14 \mathrm{TeV}$ & 3.08 & 1.33 \\
\hline \multirow{2}{*}{ BP2 } & $\sqrt{s}=7 \mathrm{TeV}$ & 3.39 & 2.13 \\
\hline & $\sqrt{s}=14 \mathrm{TeV}$ & 3.90 & 2.14 \\
BP3 & $\sqrt{s}=7 \mathrm{TeV}$ & 4.35 & 1.71 \\
\hline & $\sqrt{s}=14 \mathrm{TeV}$ & 4.39 & 1.90 \\
$\mathrm{BP} 4$ & $\sqrt{s}=7 \mathrm{TeV}$ & 5.24 & 1.86 \\
\hline
\end{tabular}

Table 8: The ratios $\frac{\sigma\left(p p \rightarrow e \mu \mu+E_{T}\right)}{\sigma\left(p p \rightarrow 3 \ell+E_{T}\right)}$ and $\frac{\sigma\left(p p \rightarrow e e e+E_{T}\right)}{\sigma\left(p p \rightarrow 3 \ell+E_{T}\right)}$ for the four benchmark points.

\section{Conclusion}

We consider the minimal supersymmetric inverse seesaw model and study its characteristic signatures at the LHC. This model, with only one pair of singlet superfields explains existing neutrino oscillation data. The model is rich from phenomenological point of view and can lead to potentially testable signatures at the hadron colliders. In this R-parity conserving model, one of the singlet sneutrino (with a small admixture of the doublet sneutrino) is the lightest supersymmetric particle (LSP) and as a result shows up in the collider as missing energy. Charginos can decay to charged leptons plus singlet sneutrino LSP. The decay patterns of the chargino are controlled by the same parameters which generate the neutrino mixing angles.

In order to study this correlation of the chargino decays and the neutrino mixing angles, we look at specific trilepton $+\mathbb{E}_{T}$ signatures at the LHC. We show that the ratios of cross sections of this studied trilepton $+\mathbb{E}_{T}$ final states in certain flavour specific channels $\left(\mu e e+\mathbb{E}_{T}, \mu \mu \mu+\mathbb{E}_{T}, \tau e e+\mathbb{E}_{T}, \tau \mu \mu+\mathbb{E}_{T}\right)$ nicely correlate with the atmospheric neutrino mixing angle. We explore different points in the parameter space to study this correlation. A measurement of these cross sections thus provide an interesting test of the minimal supersymmetric inverse seesaw model. The hard missing $E_{T}$ spectrum makes this trilepton final state statistically significant by reducing certain standard model background events significantly. We adhere to different cuts to reduce the backgrounds coming from some other channels. Motivated by the recent results from the ATLAS and the CMS experiments, we work in a scenario with heavy squarks and gluinos and a relatively light electroweak sector. The results of our analysis suggest that the theoretical parameter space of this model can be constrained by the data collected at the LHC with center-of-mass energy $7 \mathrm{TeV}$ and for an integrated luminosity of $25 \mathrm{fb}^{-1}$. On the other hand, a measured value of this ratio at the LHC can give us an alternative estimate of $\tan ^{2} \theta_{23}$ and confirm (or rule out) this minimal supersymmetric inverse seesaw model as a possible explanation of neutrino masses and mixing. We also show, as a distinct feature of this model, the cross sections of $p p \rightarrow e \mu \mu+\mathbb{E}_{T}$ and $p p \rightarrow e e e+\mathbb{E}_{T}$ are suppressed compared to the total chosen trilepton $+\mathbb{E}_{T}$ cross section because of the restrictions on the neutrino reactor angle imposed by neutrino data. 


\section{Acknowledgments}

SM wishes to thank the Department of Science and Technology, Government of India for a Senior Research Fellowship. SB would like to thank the Department of Theoretical Physics, IACS for the hospitality while this work was being proposed and initiated. SB would also like to thank PhD program of HRI and RECAPP, HRI for hospitality and financial support during the initial phase of this work. PG would like to thank the Council of Scientific and Industrial Research, Government of India for the financial assistance during the initial phase of this work. PG's work is supported by the Spanish MICINN under grant FPA2009-08958. PG also thank the support of the MICINN under the ConsoliderIngenio 2010 Programme with grant MultiDark CSD2009-00064, the Community of Madrid under grant HEPHACOS S2009/ESP-1473, and the European Union under the Marie Curie-ITN program PITN-GA-2009-237920. PG and SM wish to thank RECAPP, HRI for hospitality during a part of the investigation. We are very grateful to Asesh Krishna Datta for some very helpful discussions. We also thank Nabanita Bhattacharyya, Anindya Datta, Martin Hirsch, Partha Konar, Satyanarayan Mukhopadhyay and Sujoy Poddar for many useful comments and suggestions. PG is grateful to Chan Beom Park for his insightful suggestions. SR acknowledges the hospitality provided by the Helsinki Institute of Physics and the CERN Theory Group during the final phase of this work.

\section{References}

[1] T. Schwetz, M. A. Tortola, and J. W. F. Valle, Three-flavour neutrino oscillation update, New J. Phys. 10 (2008) 113011, 0808.2016.

[2] M. C. Gonzalez-Garcia, M. Maltoni, and J. Salvado, Updated global fit to three neutrino mixing: status of the hints of $\theta_{13}>0$, JHEP 04 (2010) 056, 1001.4524].

[3] T. Schwetz, M. Tortola, and J. W. F. Valle, Global neutrino data and recent reactor fluxes: status of three-flavour oscillation parameters, New J. Phys. 13 (2011) 063004, 1103.0734.

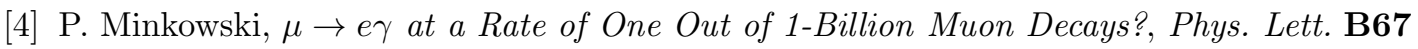
(1977) 421.

[5] T. Yanagida, Horizontal gauge symmetry and masses of neutrinos, . In Proceedings of the Workshop on the Baryon Number of the Universe and Unified Theories, Tsukuba, Japan, 13-14 Feb 1979.

[6] R. N. Mohapatra and G. Senjanovic, Neutrino mass and spontaneous parity nonconservation, Phys. Rev. Lett. 44 (1980) 912.

[7] S. L. Glashow, The future of elementary particle physics, NATO Adv. Study Inst. Ser. B Phys. 59 (1980) 687.

[8] M. Gell-Mann, P. Ramond, and R. Slansky, Complex spinors and unified theories, . Print-80-0576 (CERN).

[9] J. Schechter and J. W. F. Valle, Neutrino Masses in SU(2) $\times U(1)$ Theories, Phys. Rev. D22 (1980) 2227.

[10] J. Schechter and J. W. F. Valle, Neutrino Decay and Spontaneous Violation of Lepton Number, Phys. Rev. D25 (1982) 774. 
[11] S. Weinberg, Baryon and Lepton Nonconserving Processes, Phys. Rev. Lett. 43 (1979) $1566-1570$.

[12] S. Weinberg, Varieties of Baryon and Lepton Nonconservation, Phys. Rev. D22 (1980) 1694.

[13] WMAP Collaboration, E. Komatsu et. al., Seven-Year Wilkinson Microwave Anisotropy Probe (WMAP) Observations: Cosmological Interpretation, Astrophys. J. Suppl. 192 (2011) $18,1001.4538$.

[14] R. N. Mohapatra, Mechanism for understanding small neutrino mass in superstring theories, Phys. Rev. Lett. 56 (1986) 561-563.

[15] R. N. Mohapatra and J. W. F. Valle, Neutrino mass and baryon-number nonconservation in superstring models, Phys. Rev. D34 (1986) 1642.

[16] M. C. Gonzalez-Garcia and J. W. F. Valle, Fast decaying neutrinos and observable flavour violation in a new class of Majoron models, Phys. Lett. B216 (1989) 360.

[17] F. Bazzocchi, D. G. Cerdeno, C. Munoz, and J. W. F. Valle, Calculable inverse-seesaw neutrino masses in supersymmetry, Phys. Rev. D81 (2010) 051701, 0907.1262.

[18] G. R. Farrar and P. Fayet, Phenomenology of the Production, Decay, and Detection of New Hadronic States Associated with Supersymmetry, Phys. Lett. B76 (1978) 575-579.

[19] S. Weinberg, Supersymmetry at Ordinary Energies. 1. Masses and Conservation Laws, Phys. Rev. D26 (1982) 287.

[20] N. Sakai and T. Yanagida, Proton Decay in a Class of Supersymmetric Grand Unified Models, Nucl. Phys. B197 (1982) 533.

[21] S. Dimopoulos, S. Raby, and F. Wilczek, Proton Decay in Supersymmetric Models, Phys. Lett. B112 (1982) 133.

[22] M. Hirsch, T. Kernreiter, J. C. Romao, and A. Villanova del Moral, Minimal Supersymmetric Inverse Seesaw: Neutrino masses, lepton flavour violation and LHC phenomenology, JHEP 01 (2010) 103, 0910.2435.

[23] M. Malinsky, T. Ohlsson, Z.-Z. Xing, and H. Zhang, Non-unitary neutrino mixing and CP violation in the minimal inverse seesaw model, Phys. Lett. B679 (2009) 242-248, 0905.2889 .

[24] F. Deppisch and J. W. F. Valle, Enhanced lepton flavour violation in the supersymmetric inverse seesaw model, Phys. Rev. D72 (2005) 036001, [hep-ph/0406040].

[25] F. Deppisch, T. S. Kosmas, and J. W. F. Valle, Enhanced $\mu^{-}-e^{-}$conversion in nuclei in the inverse seesaw model, Nucl. Phys. B752 (2006) 80-92, hep-ph/0512360.

[26] D. Ibanez, S. Morisi, and J. W. F. Valle, Inverse tri-bimaximal type-III seesaw and lepton flavor violation, Phys. Rev. D80 (2009) 053015, 0907.3109.

[27] P. S. Bhupal Dev and R. N. Mohapatra, TeV Scale Inverse Seesaw in SO(10) and Leptonic Non- Unitarity Effects, Phys. Rev. D81 (2010) 013001, 0910.3924.

[28] J. Garayoa, M. C. Gonzalez-Garcia, and N. Rius, Soft leptogenesis in the inverse seesaw model, JHEP 02 (2007) 021, hep-ph/0611311.

[29] P.-H. Gu and U. Sarkar, Leptogenesis with Linear, Inverse or Double Seesaw, Phys. Lett. B694 (2010) 226-232, [1007.2323. 
[30] S. Blanchet, P. S. Bhupal Dev, and R. N. Mohapatra, Leptogenesis with TeV Scale Inverse Seesaw in SO(10), Phys. Rev. D82 (2010) 115025, 1010.1471.

[31] C. Arina, F. Bazzocchi, N. Fornengo, J. C. Romao, and J. W. F. Valle, Minimal supergravity sneutrino dark matter and inverse seesaw neutrino masses, Phys. Rev. Lett. 101 (2008) 161802, 0806.3225.

[32] Z. Kang, J. Li, T. Li, T. Liu, and J. Yang, Asymmetric Sneutrino Dark Matter in the NMSSM with Minimal Inverse Seesaw, . arXiv:1102.5644 [hep-ph].

[33] H. An, P. S. Bhupal Dev, Y. Cai, and R. N. Mohapatra, Sneutrino Dark Matter in Gauged Inverse Seesaw Models for Neutrinos, 1110.1366 .

[34] ATLAS Collaboration, G. Aad et. al., Search for squarks and gluinos using final states with jets and missing transverse momentum with the ATLAS detector in sqrt(s) $=7 \mathrm{TeV}$ proton-proton collisions, 1109.6572 .

[35] CMS Collaboration, S. Chatrchyan et. al., Search for Supersymmetry at the LHC in Events with Jets and Missing Transverse Energy, 1109.2352.

[36] CMS Collaboration, CMS Collaboration, Search for supersymmetry in all-hadronic final states using $m_{T_{2}}$ in $7 \mathrm{TeV}$ pp collisions at the LHC, . CMS-PAS-SUS-11-005.

[37] CMS Collaboration, CMS Collaboration, Search for supersymmetry in all-hadronic events with missing energy, . CMS-PAS-SUS-11-004.

[38] B. Mukhopadhyaya, S. Roy, and F. Vissani, Correlation between neutrino oscillations and collider signals of supersymmetry in an R-parity violating model, Phys. Lett. B443 (1998) 191-195, hep-ph/9808265.

[39] E. J. Chun and J. S. Lee, Implication of Super-Kamiokande data on R-parity violation, Phys. Rev. D60 (1999) 075006, hep-ph/9811201.

[40] S. Y. Choi, E. J. Chun, S. K. Kang, and J. S. Lee, Neutrino oscillations and R-parity violating collider signals, Phys. Rev. D60 (1999) 075002, hep-ph/9903465.

[41] A. Datta, B. Mukhopadhyaya, and F. Vissani, Tevatron signatures of an R-parity violating supersymmetric theory, Phys.Lett. B492 (2000) 324-330, hep-ph/9910296.

[42] J. Romao, M. Diaz, M. Hirsch, W. Porod, and J. Valle, A Supersymmetric solution to the solar and atmospheric neutrino problems, Phys.Rev. D61 (2000) 071703, hep-ph/9907499.

[43] W. Porod, M. Hirsch, J. Romao, and J. W. F. Valle, Testing neutrino mixing at future collider experiments, Phys. Rev. D63 (2001) 115004, hep-ph/0011248.

[44] M. Hirsch and W. Porod, Neutrino properties and the decay of the lightest supersymmetric particle, Phys.Rev. D68 (2003) 115007, hep-ph/0307364.

[45] F. De Campos, O. Eboli, M. Hirsch, M. Magro, W. Porod, et. al., Probing Neutrino Oscillations in Supersymmetric Models at the Large Hadron Collider, Phys.Rev. D82 (2010) 075002, 1006.5075.

[46] M. Hirsch, A. Vicente, and W. Porod, Spontaneous R-parity violation: Lightest neutralino decays and neutrino mixing angles at future colliders, Phys.Rev. D77 (2008) 075005, 0802.2896 .

[47] P. Ghosh and S. Roy, Neutrino masses and mixing, lightest neutralino decays and a solution to the mu problem in supersymmetry, JHEP 0904 (2009) 069, 0812.0084. 
[48] A. Bartl, M. Hirsch, A. Vicente, S. Liebler, and W. Porod, LHC phenomenology of the mu nu SSM, JHEP 0905 (2009) 120, [0903.3596.

[49] P. Bandyopadhyay, P. Ghosh, and S. Roy, Unusual Higgs boson signal in R-parity violating nonminimal supersymmetric models at the LHC, Phys.Rev. D84 (2011) 115022, 1012.5762 .

[50] Super-Kamiokande Collaboration, Y. Fukuda et. al., Evidence for oscillation of atmospheric neutrinos, Phys. Rev. Lett. 81 (1998) 1562-1567, hep-ex/9807003.

[51] SNO Collaboration, Q. R. Ahmad et. al., Direct evidence for neutrino flavor transformation from neutral-current interactions in the Sudbury Neutrino Observatory, Phys. Rev. Lett. 89 (2002) 011301, nucl-ex/0204008.

[52] KamLAND Collaboration, K. Eguchi et. al., First results from KamLAND: Evidence for reactor anti- neutrino disappearance, Phys. Rev. Lett. 90 (2003) 021802, hep-ex/0212021.

[53] M. Hirsch, H. V. Klapdor-Kleingrothaus, and S. G. Kovalenko, B-L violating masses in softly broken supersymmetry, Phys. Lett. B398 (1997) 311-314, hep-ph/9701253].

[54] Y. Grossman and H. E. Haber, Sneutrino mixing phenomena, Phys. Rev. Lett. 78 (1997) 3438-3441, hep-ph/9702421.

[55] R. Barbier et. al., R-parity violating supersymmetry, Phys. Rept. 420 (2005) 1-202, hep-ph/0406039.

[56] L. J. Hall and M. Suzuki, Explicit R-Parity Breaking in Supersymmetric Models, Nucl. Phys. B231 (1984) 419.

[57] I.-H. Lee, Lepton number violation in softly broken supersymmetry, Phys. Lett. B138 (1984) 121.

[58] I.-H. Lee, Lepton Number Violation in Softly Broken Supersymmetry. 2, Nucl. Phys. B246 (1984) 120.

[59] R. Hempfling, Neutrino Masses and Mixing Angles in SUSY-GUT Theories with explicit R-Parity Breaking, Nucl. Phys. B478 (1996) 3-30, hep-ph/9511288.

[60] Y. Grossman and H. E. Haber, Neutrino masses and sneutrino mixing in R-parity violating supersymmetry, . hep-ph/9906310.

[61] M. Hirsch, M. A. Diaz, W. Porod, J. C. Romao, and J. W. F. Valle, Neutrino masses and mixings from supersymmetry with bilinear R-parity violation: A theory for solar and atmospheric neutrino oscillations, Phys. Rev. D62 (2000) 113008, hep-ph/0004115.

[62] S. Davidson and M. Losada, Neutrino masses in the $R_{p}$ violating MSSM, JHEP 05 (2000) 021, hep-ph/0005080.

[63] A. Abada, S. Davidson, and M. Losada, Neutrino masses and mixings in the MSSM with soft bilinear R(p) violation, Phys. Rev. D65 (2002) 075010, hep-ph/0111332.

[64] Y. Grossman and S. Rakshit, Neutrino masses in R-parity violating supersymmetric models, Phys. Rev. D69 (2004) 093002, hep-ph/0311310.

[65] E. Ma, Radiative inverse seesaw mechanism for nonzero neutrino mass, Phys. Rev. D80 (2009) 013013, 0904.4450.

[66] M. Frank and H. N. Saif, Trilepton signals from chargino - neutralino production at the CERN p p collider in a supersymmetric left-right model, J. Phys. G22 (1996) 1653-1660. 
[67] Do Collaboration, B. Abbott et. al., Search for the trilepton signature from the associated production of SUSY $\chi_{1}^{ \pm} \chi_{2}^{0}$ gauginos, Phys. Rev. Lett. 80 (1998) 1591-1596, hep-ex/9705015.

[68] V. D. Barger, C. Kao, and T.-j. Li, Trilepton signal of minimal supergravity at the Tevatron including tau lepton contributions, Phys. Lett. B433 (1998) 328-334, hep-ph/9804451.

[69] V. D. Barger and C. Kao, Trilepton signature of minimal supergravity at the upgraded Tevatron, Phys. Rev. D60 (1999) 115015, hep-ph/9811489.

[70] K. T. Matchev and D. M. Pierce, Supersymmetry reach of the Tevatron via trilepton, like sign dilepton and dilepton plus tau jet signatures, Phys. Rev. D60 (1999) 075004, hep-ph/9904282.

[71] H. Baer, M. Drees, F. Paige, P. Quintana, and X. Tata, Trilepton signal for supersymmetry at the Fermilab Tevatron revisited, Phys. Rev. D61 (2000) 095007, hep-ph/9906233.

[72] K. T. Matchev and D. M. Pierce, New backgrounds in trilepton, dilepton and dilepton plus tau jet SUSY signals at the Tevatron, Phys. Lett. B467 (1999) 225-231, hep-ph/9907505.

[73] M. Bisset, F. Moortgat, and S. Moretti, Trilepton + top signal from chargino neutralino decays of MSSM charged Higgs bosons at the LHC, Eur. Phys. J. C30 (2003) 419-434, hep-ph/0303093.

[74] Z. Sullivan and E. L. Berger, Trilepton production at the CERN LHC: Standard model sources and beyond, Phys. Rev. D78 (2008) 034030, 0805.3720.

[75] CDF Collaboration, T. Aaltonen et. al., Search for Supersymmetry in p anti-p Collisions at $\sqrt{s}=1.96-T e V$ Using the Trilepton Signature of Chargino-Neutralino Production, Phys. Rev. Lett. 101 (2008) 251801, 0808.2446.

[76] Do Collaboration, V. M. Abazov et. al., Search for associated production of charginos and neutralinos in the trilepton final state using 2.3 fb-1 of data, Phys. Lett. B680 (2009) 34-43, 0901.0646 .

[77] N. Bhattacharyya and A. Datta, Tracking down the elusive charginos / neutralinos through $\tau$ leptons at the large hadron collider, Phys. Rev. D80 (2009) 055016, 0906.1460.

[78] F. del Aguila and J. Aguilar-Saavedra, Distinguishing seesaw models at LHC with multi-lepton signals, Nucl.Phys. B813 (2009) 22-90, [0808.2468].

[79] F. del Aguila and J. Aguilar-Saavedra, Electroweak scale seesaw and heavy Dirac neutrino signals at LHC, Phys.Lett. B672 (2009) 158-165, 0809.2096.

[80] C.-Y. Chen and P. S. Bhupal Dev, Multi-Lepton Collider Signatures of Heavy Dirac and Majorana Neutrinos, 1112.6419.

[81] CMS Collaboration, G. L. Bayatian et. al., CMS physics: Technical design report, . CERN-LHCC-2006-001.

[82] G. 't Hooft, Naturalness, chiral symmetry, and spontaneous chiral symmetry breaking, NATO Adv. Study Inst. Ser. B Phys. 59 (1980) 135.

[83] H. Nunokawa, S. J. Parke, and J. W. F. Valle, CP Violation and Neutrino Oscillations, Prog. Part. Nucl. Phys. 60 (2008) 338-402, 0710.0554.

[84] Particle Data Group Collaboration, K. Nakamura et. al., Review of particle physics, J. Phys. G37 (2010) 075021. 
[85] A. Djouadi, J.-L. Kneur, and G. Moultaka, SuSpect: A Fortran code for the supersymmetric and Higgs particle spectrum in the MSSM, Comput. Phys. Commun. 176 (2007) 426-455, hep-ph/0211331.

[86] T. Sjostrand, S. Mrenna, and P. Z. Skands, PYTHIA 6.4 Physics and Manual, JHEP 05 (2006) 026, hep-ph/0603175.

[87] CTEQ Collaboration, H. L. Lai et. al., Global QCD analysis of parton structure of the nucleon: CTEQ5 parton distributions, Eur. Phys. J. C12 (2000) 375-392, hep-ph/9903282.

[88] J. Pumplin et. al., New generation of parton distributions with uncertainties from global QCD analysis, JHEP 07 (2002) 012, hep-ph/0201195.

[89] M. L. Mangano, M. Moretti, F. Piccinini, R. Pittau, and A. D. Polosa, ALPGEN, a generator for hard multiparton processes in hadronic collisions, JHEP 07 (2003) 001, hep-ph/0206293.

[90] A. J. Barr and C. Gwenlan, The Race for supersymmetry: Using m(T2) for discovery, Phys.Rev. D80 (2009) 074007, 0907.2713.

[91] The CMS Collaboration, Performance of tau reconstruction algorithms in 2010 data collected with CMS, . CMS PAS TAU-11-001.

[92] N. Bhattacharyya, A. Datta, and S. Poddar, Lepton Flavours at the Early LHC Experiments as the Footprints of the Dark Matter Producing Mechanisms, Phys. Rev. D78 (2008) 075030, 0807.0278.

[93] W. Beenakker, M. Klasen, M. Kramer, T. Plehn, M. Spira, and P. Zerwas, The Production of charginos / neutralinos and sleptons at hadron colliders, Phys.Rev.Lett. 83 (1999) 3780-3783, hep-ph/9906298.

[94] N. Kidonakis and R. Vogt, The Theoretical top quark cross section at the Tevatron and the LHC, Phys.Rev. D78 (2008) 074005, 0805.3844.

[95] M. Cacciari, S. Frixione, M. Mangano, P. Nason, and G. Ridolfi, The t anti-t cross-section at 1.8-TeV and 1.96-TeV: A Study of the systematics due to parton densities and scale dependence, JHEP 0404 (2004) 068, hep-ph/0303085.

[96] T2K Collaboration, K. Abe et. al., Indication of Electron Neutrino Appearance from an Accelerator-produced Off-axis Muon Neutrino Beam, Phys. Rev. Lett. 107 (2011) 041801, 1106.2822 .

[97] C. G. Lester and D. J. Summers, Measuring masses of semiinvisibly decaying particles pair produced at hadron colliders, Phys. Lett. B463 (1999) 99-103, hep-ph/9906349.

[98] A. Barr, C. Lester, and P. Stephens, m(T2) : The Truth behind the glamour, J. Phys. G29 (2003) 2343-2363, hep-ph/0304226.

[99] A. J. Barr, B. Gripaios, and C. G. Lester, Transverse masses and kinematic constraints: from the boundary to the crease, JHEP 11 (2009) 096, 0908.3779].

[100] P. Konar, K. Kong, K. T. Matchev, and M. Park, Dark Matter Particle Spectroscopy at the LHC: Generalizing $M_{T_{2}}$ to Asymmetric Event Topologies, JHEP 04 (2010) 086, 0911.4126.

[101] K. Agashe, D. Kim, D. G. Walker, and L. Zhu, Using $M_{T_{2}}$ to Distinguish Dark Matter Stabilization Symmetries, Phys.Rev. D84 (2011) 055020, 1012.4460.

[102] http://www.fnal.gov/pub/now/tevlum.html. 